\title{
Chandra X\#Ray Observations of 19 Millisecond Pulsars in the Globular Cluster 47 Tucanae
}

\section{Citation}

Bogdanov, Slavko, Jonathan E. Grindlay, Craig O. Heinke, Fernando Camilo, Paulo C. C. Freire, and Werner Becker. 2006. "ChandraX\#Ray Observations of 19 Millisecond Pulsars in the Globular Cluster 47 Tucanae." The Astrophysical Journal 646 (2): 1104-15. https:// doi.org/10.1086/505133.

\section{Permanent link}

http://nrs.harvard.edu/urn-3:HUL.InstRepos:41399932

\section{Terms of Use}

This article was downloaded from Harvard University's DASH repository, and is made available under the terms and conditions applicable to Other Posted Material, as set forth at http:// nrs.harvard.edu/urn-3:HUL.InstRepos:dash.current.terms-of-use\#LAA

\section{Share Your Story}

The Harvard community has made this article openly available. Please share how this access benefits you. Submit a story.

Accessibility 


\title{
CHANDRA X-RAY OBSERVATIONS OF 19 MILLISECOND PULSARS IN THE GLOBULAR CLUSTER 47 TUCANAE
}

\author{
Slavko Bogdanov, ${ }^{1}$ Jonathan E. Grindlay, ${ }^{1}$ Craig O. Heinke, ${ }^{1,2,3}$ Fernando Camilo, ${ }^{4}$ \\ Paulo C. C. Freire, ${ }^{5}$ and Werner Becker ${ }^{6}$ \\ Received 2006 January 19; accepted 2006 April 11
}

\begin{abstract}
We present spectral and long-timescale variability analyses of Chandra X-Ray Observatory ACIS-S observations of the 19 millisecond pulsars (MSPs) with precisely known positions in the globular cluster 47 Tucanae. The X-ray emission of the majority of these MSPs is well described by a thermal (blackbody or neutron star hydrogen atmosphere) spectrum with a temperature $T_{\text {eff }} \sim(1-3) \times 10^{6} \mathrm{~K}$, emission radius $R_{\text {eff }} \sim 0.1-3 \mathrm{~km}$, and luminosity $L_{\mathrm{X}} \sim 10^{30}-10^{31} \mathrm{ergs} \mathrm{s}^{-1}$. For several MSPs, there are indications that a second thermal component is required, similar to what is seen in some nearby field MSPs. The observed radiation most likely originates from the heated magnetic polar caps of the MSPs. The small apparent scatter in $L_{\mathrm{X}}$ is consistent with thermal emission from the polar caps of a global dipole field, although the small emission areas may imply either a more complex small-scale magnetic field configuration near the neutron star surface or nonuniform polar cap heating. The radio eclipsing binary MSPs 47 Tuc J, O, and W show a significant nonthermal (power-law) component, with spectral photon index $\Gamma \sim 1-1.5$, which most likely originates in an intrabinary shock formed due to interaction between the relativistic pulsar wind and matter from the stellar companion. We reexamine the X-ray-spin-down luminosity relation $\left(L_{\mathrm{X}}-\dot{E}\right.$ relation) and find that for the MSPs with thermal spectra $L_{\mathrm{X}} \propto \dot{E}^{\beta}$, where $\beta \sim 0.2 \pm 1.1$. Due to the large uncertainties in both parameters, the result is consistent with both the linear $L_{\mathrm{X}}-\dot{E}$ relation and the flatter $L_{\mathrm{X}} \propto \dot{E}^{0.5}$ predicted by polar cap heating models. In terms of X-ray properties, we find no clear systematic differences between MSPs in globular clusters and in the field of the Galaxy. We discuss the implications of these results on the present understanding of the X-ray emission properties of MSPs.
\end{abstract}

Subject headings: globular clusters: general — globular clusters: individual (47 Tucanae) — pulsars: general — stars: neutron - X-rays: stars

Online material: extended figure

\section{INTRODUCTION}

Millisecond pulsars (MSPs) represent a distinct population of rotation-powered pulsars, characterized by short spin periods, $P \lesssim 25 \mathrm{~ms}$, and small intrinsic spin-down rates, $\dot{P}_{i} \sim 10^{-20}$, implying relatively low surface magnetic dipole field strengths $B_{\text {surf }} \propto\left(P \dot{P}_{i}\right)^{1 / 2} \sim 10^{8}-10^{10} \mathrm{G}$ and large characteristic spin-down ages $\tau \equiv P / 2 \dot{P}_{i} \gtrsim 1$ Gyr. These objects have been studied extensively at radio wavelengths since their discovery (Backer et al. 1982), but at X-ray energies, where the bulk of observable radiation is expected, this has become possible only relatively recently, owing to their intrinsic faintness $\left(L_{\mathrm{X}}<10^{33} \mathrm{ergs} \mathrm{s}^{-1}\right)$. The ROSAT (Röntgensatellit) observatory was the first to detect $\mathrm{X}$-ray emission from MSPs, including 10 in the field of the Galaxy and one in the globular cluster M28 (see Becker \& Trümper 1999 and references therein). In recent years, the unprecedented sensitivity and spatial resolution of the Chandra X-Ray Observatory have allowed the detection of X-ray counterparts to radio

\footnotetext{
${ }^{1}$ Harvard-Smithsonian Center for Astrophysics, 60 Garden Street, Cambridge, MA 02138; sbogdanov@cfa.harvard.edu, josh@cfa.harvard.edu, cheinke@ cfa.harvard.edu.

${ }^{2}$ Northwestern University, Department of Physics and Astronomy, 2145 Sheridan Road, Evanston, IL 60208; cheinke@northwestern.edu.

${ }^{3}$ Lindheimer Postdoctoral Fellow.

${ }^{4}$ Columbia Astrophysics Laboratory, Columbia University, 550 West 120th Street, New York, NY 10027; fernando@astro.columbia.edu.

5 National Astronomy and Ionosphere Center, Arecibo Observatory, HC3 Box 53995, PR 00612; pfreire@naic.edu.

6 Max-Planck-Institut für Extraterrestrische Physik, D-85740 Garching bei München, Germany; web@mpe.mpg.de.
}

MSPs in several globular clusters: 47 Tucanae and NGC 6397 (Grindlay et al. 2002), NGC 6752 (D'Amico et al. 2002), M4 (Bassa et al. 2004), possibly M30 (Ransom et al. 2004), and M71 (R. F. Elsner et al. 2006, in preparation). In addition, the Chandra and XMM-Newton telescopes have allowed much more detailed studies of the existing sample of X-ray-detected MSPs than were previously possible (Zavlin et al. 2002; Becker \& Aschenbach 2002; Becker et al. 2003; Webb et al. 2004a, 2004b).

The X-ray emission detected from MSPs is observed to be of both nonthermal and thermal character. In the X-ray-bright MSPs, B1821-24 and B1937+21, the narrow X-ray pulse profiles indicate that the emission is beamed and therefore must be nonthermal radiation originating in the pulsar magnetosphere (Becker \& Trümper 1999). Alternatively, as seen in the "black widow" pulsar, B1957+20, nonpulsed nonthermal X-rays can be produced by interaction of the relativistic particle wind from the pulsar with matter from a stellar companion or the interstellar medium (Stappers et al. 2003). Thermal emission observed from nearby MSPs (see, e.g., Zavlin et al. 2002; Becker $\&$ Aschenbach 2002) is believed to originate from the magnetic polar caps of the underlying neutron star (NS), heated by a backflow of energetic particles from the pulsar magnetosphere (see, e.g., Harding \& Muslimov 2002; Zhang \& Cheng 2003 and references therein).

The detection of 16 MSPs in the globular cluster 47 Tucanae (NGC 104, hereafter 47 Tuc) by the Chandra ACIS-I (Advanced CCD Imaging Spectrometer-Imager) instrument has granted us an excellent opportunity for a detailed study of the physical 


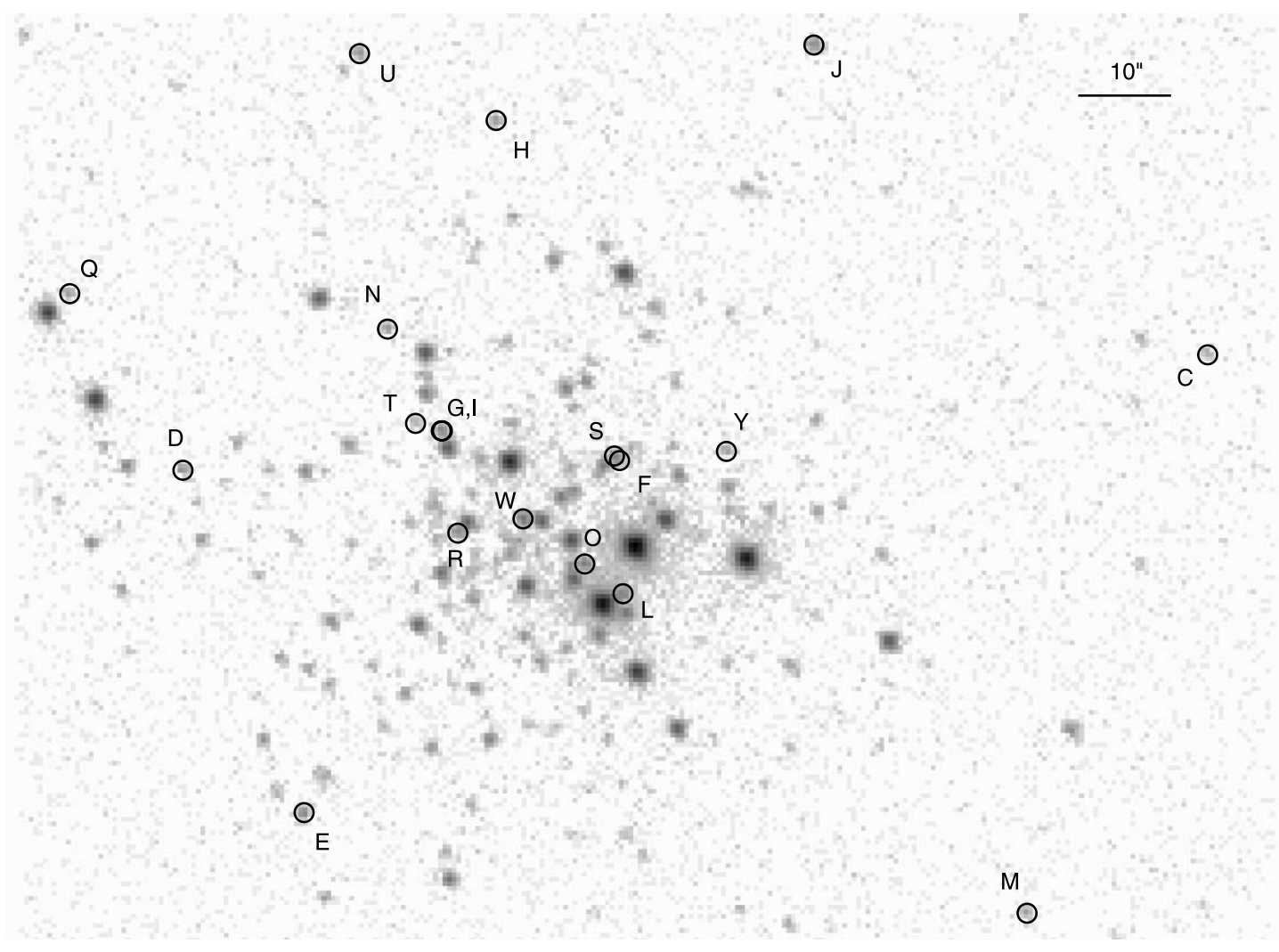

FIg. 1.- Merged 0.3-6 keV image of all four Chandra ACIS-S observations of 47 Tucanae showing the positions of the X-ray counterparts of 19 millisecond pulsars. The $1^{\prime \prime}$ circles are centered on the radio MSP locations and enclose $\geq 90 \%$ of the total energy for $0.5-1 \mathrm{keV}$ thermal sources. The gray scale corresponds to number of counts increasing logarithmically from zero (white) to 8306 (black). North is up, and east is to the left.

properties of this class of objects (Grindlay et al. 2001, 2002). These pulsars were discovered at radio wavelengths by Manchester et al. (1990, 1991), Robinson et al. (1995), and Camilo et al. (2000). With further pulse timing observations, Freire et al. (2001a, 2003) were able to obtain precise positions for 16 of the 22 MSPs currently known in this globular cluster (Camilo \& Rasio 2005). In addition, Edmonds et al. (2002) detected the optical counterpart of 47 Tuc $\mathrm{W}$, which does not have an accurate radio timing position, by matching the binary phase and period of an optical candidate with that of the radio pulsar. Finally, timing solutions have recently been obtained for 47 Tuc R and Y (P. C. Freire et al. 2006, in preparation), thus increasing the number of MSPs with known positions in this cluster to 19. Grindlay et al. (2002) have reported in detail on the analysis of the X-ray emission properties of the 47 Tuc MSPs, using the Chandra ACIS-I observations mentioned above. However, due to the inherent faintness of these sources $\left(L_{\mathrm{X}} \sim 10^{30}-10^{31} \mathrm{ergs} \mathrm{s}^{-1}\right)$ and the limited exposure time $(72 \mathrm{ks}$ of total data obtained, resulting in 1-30 detected counts for each MSP), the exact nature of the X-ray emission could not be determined with great confidence, although some interesting constraints could be placed. In the meantime, additional X-ray observations of 47 Tuc have been carried out, allowing further study of the properties of globular cluster MSPs as well as MSPs in general.

In this paper, we present spectral and long-timescale variability analyses of the X-ray counterparts of MSPs in the globular cluster 47 Tuc based on deep Chandra ACIS-S observations. This paper extends the work presented in Heinke et al. (2005). The work is organized in the following manner: $\S 2$ describes the observations and data reduction procedure; in $\S 3$ we discuss the X-ray spectral properties of the MSPs, while in $\S 4$ we investigate the long-term temporal behavior of the X-ray emission, and in $\S 5$ we compare the X-ray and radio pulsar properties. Finally, in $\S 6$ we discuss the implications of the results presented, and we offer conclusions in $\S 7$.

\section{OBSERVATIONS AND DATA REDUCTION}

The data set presented here consists of four 65 ks observations of the core of the globular cluster 47 Tuc with the S3 chip of the Chandra ACIS-S detector at the focus. The first three observations were performed between 2002 September 29 and October 3, while the fourth was carried out on 2002 October 11. These observations have resulted in the detection of $\sim 300$ X-ray sources within the half-mass radius of 47 Tuc, including X-ray counterparts to all of the 19 MSPs with known positions. In this present paper we focus on the counterparts of the radio MSPs; for a comprehensive analysis of the other X-ray sources, see Heinke et al. (2005). The initial data reduction and image processing were performed using the $\mathrm{CIAO}^{7} 3.0$ software package and are also described in detail by Heinke et al. (2005). Figure 1 shows the combined image of all four observations with $1^{\prime \prime}$ circles centered at the radio position of each MSP. The CIAO tool wavdetect was able to detect an X-ray source at the position of each of the radio MSPs, including 47 Tuc C, L, M, Q, and T, which were not detected or only marginally detected in the 2000 ACIS-I observations (Grindlay et al. 2002). The MSPs 47 Tuc G and I, separated by just 0 "' 12 , could not be individually resolved, whereas 47 Tuc $F$ and $S$, separated by 0.7 , were again identified as a single elongated source. In this paper, we also include analyses

\footnotetext{
${ }^{7}$ Chandra Interactive Analysis of Observations (http://asc.harvard.edu/ciao).
} 
TABLE 1

Net Counts from the 19 MSPs in 47 Tuc

\begin{tabular}{|c|c|c|c|c|}
\hline MSP & $\begin{array}{l}\text { Soft Counts } \\
(0.3-0.6 \mathrm{keV})\end{array}$ & $\begin{array}{l}\text { Medium Counts } \\
(0.6-1.5 \mathrm{keV})\end{array}$ & $\begin{array}{l}\text { Hard Counts } \\
(1.5-4 \mathrm{keV})\end{array}$ & $\begin{array}{l}\text { Total Counts } \\
(0.3-4 \mathrm{keV})\end{array}$ \\
\hline С..... & $12 \pm 4$ & $29 \pm 5$ & $4 \pm 2$ & $45 \pm 7$ \\
\hline D.................... & $20 \pm 5$ & $60 \pm 8$ & $12 \pm 4$ & $92 \pm 10$ \\
\hline E... & $39 \pm 6$ & $74 \pm 9$ & $11 \pm 3$ & $124 \pm 11$ \\
\hline $\mathrm{F}+\mathrm{S}^{\mathrm{a}} .$. & $67 \pm 8$ & $159 \pm 13$ & $20 \pm 5$ & $246 \pm 16$ \\
\hline $\mathrm{F}^{\mathrm{a}}$. & $15 \pm 12$ & $83 \pm 21$ & $12 \pm 7$ & $93 \pm 18$ \\
\hline $\mathrm{G}+\mathrm{I} \ldots \ldots \ldots$ & $40 \pm 6$ & $102 \pm 10$ & $16 \pm 4$ & $158 \pm 12$ \\
\hline 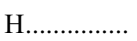 & $24 \pm 5$ & $46 \pm 7$ & $2 \pm 2$ & $72 \pm 9$ \\
\hline J .................... & $25 \pm 5$ & $80 \pm 9$ & $32 \pm 6$ & $137 \pm 12$ \\
\hline ......... & $37 \pm 8$ & $132 \pm 14$ & $11 \pm 7$ & $180 \pm 18$ \\
\hline M............... & $7 \pm 3$ & $47 \pm 7$ & $2 \pm 2$ & $56 \pm 8$ \\
\hline N.................... & $20 \pm 5$ & $40 \pm 7$ & $10 \pm 3$ & $70 \pm 9$ \\
\hline O..................... & $30 \pm 6$ & $61 \pm 9$ & $10 \pm 4$ & $101 \pm 12$ \\
\hline Q.................. & $18 \pm 4$ & $40 \pm 6$ & $9 \pm 3$ & $67 \pm 8$ \\
\hline R... & $41 \pm 8$ & $105 \pm 11$ & $16 \pm 5$ & $162 \pm 14$ \\
\hline$S^{a}$. & $52 \pm 12$ & $76 \pm 21$ & $8 \pm 7$ & $153 \pm 20$ \\
\hline Т .................. & $10 \pm 3$ & $22 \pm 5$ & $6 \pm 3$ & $38 \pm 7$ \\
\hline U..................... & $10 \pm 3$ & $67 \pm 8$ & $11 \pm 3$ & $88 \pm 9$ \\
\hline 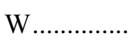 & $60 \pm 8$ & $160 \pm 13$ & $86 \pm 9$ & $306 \pm 18$ \\
\hline Y .................... & $16 \pm 5$ & $41 \pm 7$ & $5 \pm 3$ & $62 \pm 9$ \\
\hline
\end{tabular}

${ }^{\text {a }}$ For $\mathrm{F}$ and $\mathrm{S}$, we quote the combined counts as well as the counts for each MSP apportioned using the procedure described in the text.

of MSP W, whose optical counterpart was found by Edmonds et al. (2002) to coincide with the X-ray source W29 (Grindlay et al. 2001), and the MSPs R and Y, whose recently obtained radio timing solutions (P. C. Freire et al. 2006, in preparation) place them at the positions of the X-ray sources W198 and W82, respectively.

To facilitate the spectral and variability analyses, the counts for each MSP in the 0.3-8 keV energy band were extracted from circular regions with $1^{\prime \prime}$ radii around the radio MSP positions. These circles enclose $\geq 90 \%$ of the total energy for $0.5-1 \mathrm{keV}$ thermal sources. A slightly more involved extraction method was required for 47 Tuc $\mathrm{F}$ and $\mathrm{S}$, whose extraction circles overlap, as is evident in Figure 1. The relative contribution of counts from each MSP was determined by maximum likelihood fits of twodimensional Gaussians to the five X-ray sources in the vicinity of the radio positions of $\mathrm{F}$ and $\mathrm{S}$ using the Sherpa application in CIAO. This procedure was carried out in the $0.3-4 \mathrm{keV}$ range, as well as in three narrow bands $(0.3-0.8,0.8-1.5$, and $1.5-4 \mathrm{keV})$ to allow an approximate spectral analysis. Using this method we find that $62 \% \pm 7 \%$ of the total counts can be attributed to $S$. For 47 Tuc G and I, this was not possible, as their radio positions are only 0.12 apart. However, the count rate observed from their position is roughly twice that of a typical MSP in the sample and is thus consistent with being combined emission from both MSPs. The net counts for most MSPs were obtained by subtracting the background rate, obtained from three source-free regions on the image, from the total.

In general, the background noise in the energy range of interest was found to be very low $(\sim 1$ background count per pixel over $\sim 260 \mathrm{ks}$ of total exposure time). This was not the case for 47 Tuc $\mathrm{L}$, which is located very near the bright $\mathrm{X}$-ray source $\mathrm{X} 9$ (Grindlay et al. 2001). The background for this MSP was obtained by considering a section of an annulus of width $2^{\prime \prime}$ around the positions of $\mathrm{X} 9$, at the distance to $\mathrm{L}$, while excluding the $1^{\prime \prime}$ extraction circle around the MSP. Using this background region we find that $\sim 30 \%$ of the gross number of counts is due to $\mathrm{X} 9$. For 47 Tuc R, situated 1"3 from the bright source W24, this procedure was not possible due to a combination of the smaller

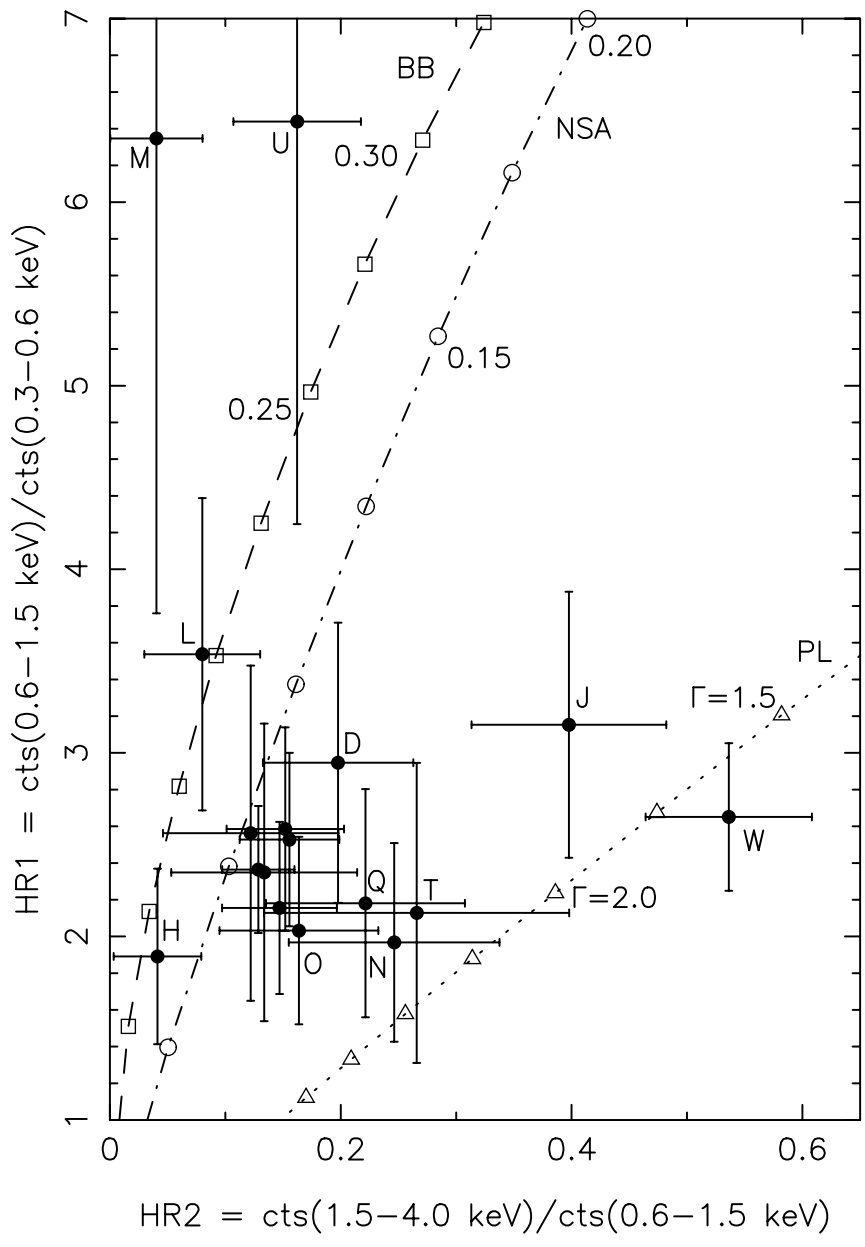

FIG. 2.-X-ray color-color diagram for the MSPs in 47 Tuc. The lines represent model tracks for absorbed power law (dotted line), blackbody (dashed line), and neutron star hydrogen atmosphere (dot-dashed line) spectra convolved with the ACIS-S instrument response. The open symbols mark values for the model tracks in intervals of $0.025 \mathrm{keV}$ for the blackbody (squares) and hydrogen atmosphere (circles) temperatures and 0.25 for the power-law photon index (triangles).

distance to W24 and the large pixel size of the ACIS detector. However, the distribution of counts within the $1^{\prime \prime}$ extraction region of $\mathrm{R}$ suggests that $\mathrm{W} 24$ contributes no more than $10 \%$ to the total counts. In the case of MSP O, the degree of contamination is difficult to gauge due to severe crowding. In addition, the X-ray counterpart of 47 Tuc $\mathrm{O}, \mathrm{W} 39$, is very likely a blend of the MSP and a variable source (see $\S 4$ ).

\section{SPECTRAL ANALYSIS}

To get a general sense of the X-ray properties of the MSPs, we have computed the hardness ratios HR1 = (medium counts) $/$ (soft counts) and HR2 = (hard counts)/(medium counts) for each object and constructed an X-ray color-color diagram. The soft $(0.3-0.6 \mathrm{keV})$, medium $(0.6-1.5 \mathrm{keV})$, and hard $(1.5-4 \mathrm{keV})$ energy bands were selected so as to reduce the errors in HR2, allowing us to discriminate between the two favored spectral models: thermal or nonthermal (power law). The net counts in the three bands for each MSP are listed in Table 1, while the resulting X-ray color-color diagram is shown in Figure 2. For comparison, we have also plotted values of HR1 and HR2 for simple absorbed models of blackbody, neutron star hydrogen atmosphere (Romani 1987; Zavlin et al. 1996; Lloyd 2003), and 
TABLE 2

Best-Fit Spectral Models and Unabsorbed Fluxes for the 47 Tuc MSPs

\begin{tabular}{|c|c|c|c|c|c|c|}
\hline MSP & Spectral Model ${ }^{\mathrm{a}}$ & $\begin{array}{c}T_{\text {eff }} \\
\left(10^{6} \mathrm{~K}\right)\end{array}$ & $\begin{array}{l}R_{\mathrm{eff}}^{\mathrm{b}} \\
(\mathrm{km})\end{array}$ & Photon Index & $\chi_{\nu}^{2} / \mathrm{dof}$ & $\begin{array}{c}F_{\mathrm{X}}(0.3-8 \mathrm{keV}) \\
\left(10^{-15} \mathrm{ergs}^{-2} \mathrm{~s}^{-1}\right)\end{array}$ \\
\hline$\ldots \ldots$. & BB & $2.02 \pm 0.18$ & $0.11 \pm 0.08$ & $\ldots$ & $0.29 / 3$ & $0.71_{-0.26}^{+0.14}$ \\
\hline $\mathrm{D}$ & $\mathrm{BB}$ & $2.20 \pm 0.17$ & $0.13 \pm 0.06$ & $\ldots$ & $1.96 / 8$ & $1.37_{-0.35}^{+0.18}$ \\
\hline 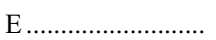 & $\mathrm{BB}$ & $1.75 \pm 0.09$ & $0.28 \pm 0.17$ & $\ldots$ & $1.60 / 6$ & $2.08_{-0.36}^{+0.26}$ \\
\hline $\mathrm{F}+\mathrm{S} \ldots \ldots \ldots \ldots \ldots \ldots$ & $\mathrm{BB}$ & $2.19 \pm 0.09$ & $0.22 \pm 0.11$ & $\ldots$ & $1.05 / 10$ & $3.80_{-0.56}^{+0.36}$ \\
\hline $\mathrm{G}+\mathrm{I} \ldots \ldots \ldots \ldots \ldots \ldots$ & $\mathrm{BB}$ & $2.21 \pm 0.12$ & $0.18 \pm 0.10$ & $\ldots$ & $0.96 / 12$ & $2.54_{-0.48}^{0.19+}$ \\
\hline 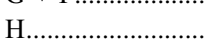 & $\mathrm{BB}$ & $1.93 \pm 0.13$ & $0.17 \pm 0.11$ & $\cdots$ & $0.55 / 6$ & $1.30_{-0.33}^{+0.48}$ \\
\hline $\mathrm{J}$ & $\mathrm{BB}+\mathrm{PL}$ & $1.73 \pm 0.21$ & $0.22 \pm 0.17$ & $1.00 \pm 0.56$ & $1.07 / 5$ & $4.77_{-1.51}^{+0.95}\left(3.28_{-2.32}^{+0.19}\right)$ \\
\hline $\mathrm{L}^{\mathrm{c}} \ldots \ldots \ldots \ldots \ldots \ldots \ldots \ldots$ & BB & $2.27 \pm 0.10$ & $0.20 \pm 0.10$ & $\ldots$ & $2.04 / 11$ & $3.54_{-0.41}^{+0.32}$ \\
\hline $\mathrm{M}$ & $\mathrm{BB}$ & $2.22 \pm 0.18$ & $0.11 \pm 0.07$ & $\ldots$ & $0.40 / 4$ & $1.01_{-0.30}^{+0.14}$ \\
\hline $\mathrm{N}$ & $\mathrm{BB}$ & $2.07 \pm 0.18$ & $0.13 \pm 0.09$ & $\ldots$ & $1.94 / 5$ & $0.98_{-0.30}^{+0.17}$ \\
\hline $\mathrm{O}^{\mathrm{c}}$ & $\mathrm{BB}+\mathrm{PL}$ & $1.76 \pm 0.15$ & $0.28 \pm 0.18$ & $1.33 \pm 0.79$ & $1.21 / 10$ & $4.44_{-0.86}^{+1.44}\left(2.23_{-1.73}^{+0.24}\right)$ \\
\hline $\mathrm{Q}$ & $\mathrm{BB}$ & $2.24 \pm 0.20$ & $0.11 \pm 0.07$ & $\ldots$ & $1.17 / 5$ & $1.00_{-0.30}^{+0.13}$ \\
\hline $\mathrm{R}$ & BB & $2.51 \pm 0.16$ & $0.15 \pm 0.08$ & $\ldots$ & $2.54 / 7$ & $2.87_{-0.56}^{+0.17}$ \\
\hline $\mathrm{T}$ & $\mathrm{BB}$ & $1.56 \pm 0.16$ & $0.19 \pm 0.17$ & $\ldots$ & $2.09 / 2$ & $0.63_{-0.26}^{+0.13}$ \\
\hline 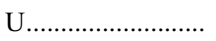 & BB & $2.76 \pm 0.22$ & $0.08 \pm 0.06$ & $\ldots$ & $0.55 / 6$ & $1.32_{-0.33}^{+0.12}$ \\
\hline W & $\mathrm{BB}+\mathrm{PL}$ & $1.52 \pm 0.28$ & $0.29 \pm 0.29$ & $1.36 \pm 0.24$ & $1.22 / 14$ & $10.90_{-2.61}^{+0.40}\left(9.55_{-1.44}^{+1.75}\right)$ \\
\hline $\mathrm{Y}$ & $\mathrm{BB}$ & $2.24 \pm 0.18$ & $0.11 \pm 0.07$ & $\ldots$ & $1.16 / 4$ & $1.03_{-0.28}^{+0.10}$ \\
\hline
\end{tabular}

${ }^{\text {a }} \mathrm{PL}$ is a power law and BB is a single-temperature blackbody model. For all MSPs the hydrogen column density was fixed at $N_{\mathrm{H}}=$ $(1.3 \pm 0.3) \times 10^{20} \mathrm{~cm}^{-2}$. In the last column, the values in parentheses for $\mathrm{J}, \mathrm{O}$, and $\mathrm{W}$ represent the flux in the PL component. All uncertainties quoted are $1 \sigma$.

${ }^{\mathrm{b}} R_{\mathrm{eff}}$ calculated assuming a distance of $4.5 \mathrm{kpc}$.

${ }^{\mathrm{c}}$ Spectrum may be contaminated by background/neighboring source(s).

power-law spectra for a range of temperatures and photon indices, respectively.

The Chandra ACIS-I observations of 47 Tuc revealed that the X-ray colors of the majority of the MSPs are consistent with those of soft, presumably thermal, sources with blackbody temperatures of $k T \sim 0.22 \mathrm{keV}$ and emission radii of $R<0.6 \mathrm{~km}$ (Grindlay et al. 2002). The X-ray color-color diagram for the ACIS-S data (Fig. 2) shows that, with the exception of 47 Tuc J and W, the MSPs are indeed soft sources, although the positions of the MSPs on the diagram are suggestive of a more complex spectrum, such as a composite thermal and nonthermal ( power-law) spectrum or a multitemperature thermal spectrum. As Grindlay et al. (2002) and Ransom et al. (2004) argue, although consistent with the X-ray colors of the MSPs, a pure thermal bremsstrahlung spectrum is ruled out, as it would require unrealistically large plasma densities, which would be inconsistent with the observed dispersion measures.

The fivefold improvement in counting statistics, resulting from the longer exposure time and the better soft response of the ACIS-S compared to the ACIS-I observations, permits a more precise determination of the nature of the X-ray emission via spectral fitting for the 47 Tuc MSPs. For this purpose, we used the total counts extracted for each MSP from the four observations to generate source and background spectra, along with the corresponding response matrix and auxiliary files. The MSP pairs 47 Tuc F and S, and G and I, were treated as single sources, as the individual spectra could not be separated. For the fainter MSPs, the extracted counts were grouped into energy bins containing at least 10 counts, while for the brighter MSPs each bin contained at least 15 counts. The subsequent least-squares fits to the spectra were performed using the $\mathrm{XSPEC}^{8}$ software package and were restricted to photon energies between 0.3 and $8 \mathrm{keV}$. In the fitting process, we considered the following spectral models as plausible physical descriptions of the MSP emission: a pure

\footnotetext{
${ }^{8}$ See http://heasarc.gsfc.nasa.gov/docs/xanadu/xspec.
}

blackbody (BB), an unmagnetized neutron star hydrogen atmosphere (NSA; Lloyd 2003), a pure power law (PL), a composite thermal and power law (BB + PL or NSA + PL), and a twotemperature thermal model (BB + BB or NSA + NSA). Throughout this analysis the hydrogen column density was fixed at the nominal value for $47 \mathrm{Tuc}, N_{\mathrm{H}}=(1.3 \pm 0.3) \times 10^{20} \mathrm{~cm}^{-2}$ (Gratton et al. 2003; Predehl \& Schmitt 1995; Cardelli et al. 1989). For the NSA model we assumed a value of $z_{g}=\left[1-\left(2 G M_{\mathrm{NS}}\right) /\right.$ $\left.\left(c^{2} R_{\mathrm{NS}}\right)\right]^{-1 / 2}-1=0.31$ for the gravitational redshift at the NS surface, appropriate for a $M_{\mathrm{NS}}=1.4 M_{\odot}, R_{\mathrm{NS}}=10 \mathrm{~km}$ star. Thus, the only free parameters for the BB and NSA fits were the effective temperature and BB/NSA normalization, while for the PL model the free parameters were the photon index and the normalization.

From the spectral fits we find that many of the 47 Tuc MSP spectra are well described by a purely thermal spectrum (BB or NSA). For the BB model the fits yielded effective temperatures and radii of $T_{\text {eff }} \sim(1.5-3.0) \times 10^{6} \mathrm{~K}$ and $R_{\text {eff }} \sim 0.1-0.5 \mathrm{~km}$, respectively, while in the case of the NSA model we obtained $T_{\text {eff }} \sim(0.8-1.8) \times 10^{6} \mathrm{~K}$ and $R_{\text {eff }} \sim 0.3-3 \mathrm{~km}$. Tables 2 and 3 list the best-fit model, the corresponding values for the fitted parameters, and the unabsorbed flux for each MSP for the BB and NSA model fits, respectively. Figure 3 shows representative thermal spectra for the 47 Tuc MSPs.

It is apparent from the values of $\chi_{\nu}^{2}$ listed in Tables 2 and 3 , as well as Figure 3, that the spectra of several MSPs are poorly fit by a single-temperature model, especially at energies above $\sim 1.5 \mathrm{keV}$. In addition, we find the fits to be unacceptable for a PL or a composite PL + BB/PL + NSA model as well. Motivated by the results for the nearby MSPs J0437-4715 (Zavlin et al. 2002) and J0030+0451 (Becker \& Aschenbach 2002), we attempted fitting a two-temperature polar cap model to the brighter thermal-emitting 47 Tuc MSPs, namely, D, E, L, and R. We found that a two-temperature spectrum can account for the excess at $>1.5 \mathrm{keV}$ apparent in Figure 3, resulting in an improved fit, although the limited count statistics do not allow us to reliably constrain the emission areas. Nonetheless, if we fix the 
TABLE 3

Best-Fit Spectral Models and Unabsorbed Fluxes for the 47 Tuc MSPs

\begin{tabular}{|c|c|c|c|c|c|c|}
\hline MSP & Spectral Model ${ }^{\mathrm{a}}$ & $\begin{array}{c}T_{\text {eff }} \\
\left(10^{6} \mathrm{~K}\right)\end{array}$ & $\begin{array}{l}R_{\mathrm{eff}}^{\mathrm{b}} \\
(\mathrm{km})\end{array}$ & Photon Index & $\chi_{\nu}^{2} / \mathrm{dof}$ & $\begin{array}{c}F_{\mathrm{X}}(0.3-8 \mathrm{keV}) \\
\left(10^{-15} \mathrm{ergs} \mathrm{cm}^{-2} \mathrm{~s}^{-1}\right)\end{array}$ \\
\hline $\mathrm{C}$ & NSA & $1.12_{-0.19}^{+0.23}$ & $0.57 \pm 0.56$ & $\ldots$ & $0.68 / 3$ & $0.73_{-0.29}^{+0.09}$ \\
\hline 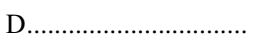 & NSA & $1.29_{-0.17}^{+0.20}$ & $0.61 \pm 0.51$ & $\ldots$ & $1.40 / 8$ & $1.54_{-0.62}^{+0.05}$ \\
\hline E & NSA & $0.88_{-0.11}^{+0.12}$ & $1.75 \pm 1.41$ & $\ldots$ & $1.03 / 6$ & $2.28_{-0.05}^{+0.02}$ \\
\hline $\mathrm{F}+\mathrm{S}$ & NSA & $1.27_{-0.09}^{+0.11}$ & $1.03 \pm 0.64$ & $\ldots$ & $0.77 / 10$ & $4.09_{-0.62}^{+0.14}$ \\
\hline $\mathrm{G}+\mathrm{I}$ & NSA & $1.28_{-0.12}^{+0.12}$ & $0.84 \pm 0.58$ & $\ldots$ & $0.60 / 12$ & $2.78_{-0.50}^{+0.16}$ \\
\hline 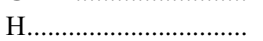 & NSA & $1.04_{-014}^{+0.16}$ & $0.94 \pm 0.79$ & $\ldots$ & $0.59 / 6$ & $1.39_{-0.68}^{+0.50}$ \\
\hline J & $\mathrm{NSA}+\mathrm{PL}$ & $0.89_{-0.16}^{+0.14}$ & $1.43 \pm 1.40$ & 1.0 & $1.00 / 6$ & $4.69_{-1.56}^{+1.07}\left(3.09_{-0.38}^{+1.99}\right)$ \\
\hline $\mathrm{L}^{\mathrm{c}}$ & NSA & $1.42_{-0.12}^{+0.16}$ & $0.78 \pm 0.50$ & $\ldots$ & $2.09 / 11$ & $3.70_{-0.72}^{+0.24}$ \\
\hline М & NSA & $\begin{array}{l}1.77_{-0.12}^{+12} \\
1.27_{-0.17}^{+0.19}\end{array}$ & $0.53 \pm 0.45$ & $\begin{array}{l}\cdots \\
\cdots\end{array}$ & $0.45 / 4$ & $\begin{array}{l}1.08_{-0.40}^{+0.72} \\
1.08\end{array}$ \\
\hline $\mathrm{N}$ & NSA & $\begin{array}{l}1.21-0.17 \\
1.20_{-0.19}^{+0.22}\end{array}$ & $0.61 \pm 0.57$ & $\begin{array}{l}\cdots \\
\cdots\end{array}$ & $1.38 / 5$ & $\begin{array}{l}1.00_{-0.40}^{+0.03} \\
1.09_{-0.50}^{+0}\end{array}$ \\
\hline 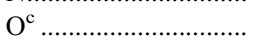 & $\mathrm{NSA}+\mathrm{PL}$ & $0.98_{-0.15}^{+0.19}$ & $1.48 \pm 1.27$ & 1.3 & $1.34 / 11$ & $4.55_{-1.49}^{+1.16}\left(1.91_{-0.17}^{+2.62}\right)$ \\
\hline Q & NSA & $1.30_{-0.21}^{+0.26}$ & $0.51 \pm 0.46$ & $\ldots$ & $0.78 / 5$ & $1.10_{-0.43}^{+0.03}$ \\
\hline 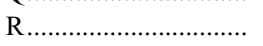 & NSA & $1.54_{-0.17}^{+0.19}$ & $0.60 \pm 0.45$ & $\ldots$ & $1.60 / 7$ & $3.19_{0.92}^{+0.13}$ \\
\hline $\mathrm{T}$ & NSA & $0.80_{-0.15}^{+0.19}$ & $0-2.4$ & $\ldots$ & $1.52 / 2$ & $0.67_{-0.32}^{+0.03}$ \\
\hline U & NSA & $1.82_{-0.24}^{+0.27}$ & $0.28 \pm 0.22$ & $\ldots$ & $0.49 / 6$ & $1.40_{-0.60}^{+0.07}$ \\
\hline 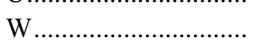 & $\mathrm{NSA}+\mathrm{PL}$ & $0.94_{-0.25}^{+0.34}$ & $0-2.1$ & $1.15 \pm 0.34$ & $1.17 / 14$ & $11.32_{-2.35}^{+2.45}\left(9.21_{-1.99}^{+2.11}\right)$ \\
\hline Y & NSA & $1.35_{-0.17}^{+0.25}$ & $0.47 \pm 0.40$ & . & $0.98 / 4$ & $1.10_{0.33}^{+0.07}$ \\
\hline
\end{tabular}

${ }^{\text {a }} \mathrm{PL}$ is a power law and NSA is a single-temperature NS hydrogen atmosphere model. For 47 Tuc $\mathrm{J}$ and $\mathrm{O}$, the PL index has been fixed at the nominal value obtained for the BB + PL fit, as the fit parameters cannot be reliably constrained for the NSA + PL with the PL index as a free parameter.

${ }^{\mathrm{b}} R_{\text {eff }}$ calculated assuming a distance of $4.5 \mathrm{kpc}$.

c Spectrum may be contaminated by background/neighboring source(s).

two temperatures to values comparable to those of J0437-4715 and $\mathrm{J} 0030+0451$, we obtain very similar results for the effective radii of the 47 Tuc MSPs to those obtained for the two field MSPs. Specifically, in the case of the NSA model, for $T_{1}=$ $2.1 \times 10^{6} \mathrm{~K}$ and $T_{2}=0.54 \times 10^{6} \mathrm{~K}$ (Zavlin et al. 2002) the fits yielded $R_{1} \sim 0.3 \mathrm{~km}$ and $R_{2} \sim 2 \mathrm{~km}$, respectively, while for a BB model with $T_{1}=3.2 \times 10^{6} \mathrm{~K}$ and $T_{2}=1.4 \times 10^{6} \mathrm{~K}($ Becker
\& Aschenbach 2002), we obtain $R_{1} \sim 50 \mathrm{~m}$ and $R_{2} \sim 300 \mathrm{~m}$. If we extend the analogy further, it seems plausible that the thermal emission from all 47 Tuc MSPs consists of two components as well. This implies that the derived values of $T_{\text {eff }}$ and $R_{\text {eff }}$ listed in Tables 2 and 3 for the single-component fit represent averages, weighted based on the relative temperatures and areas of the two emitting regions.
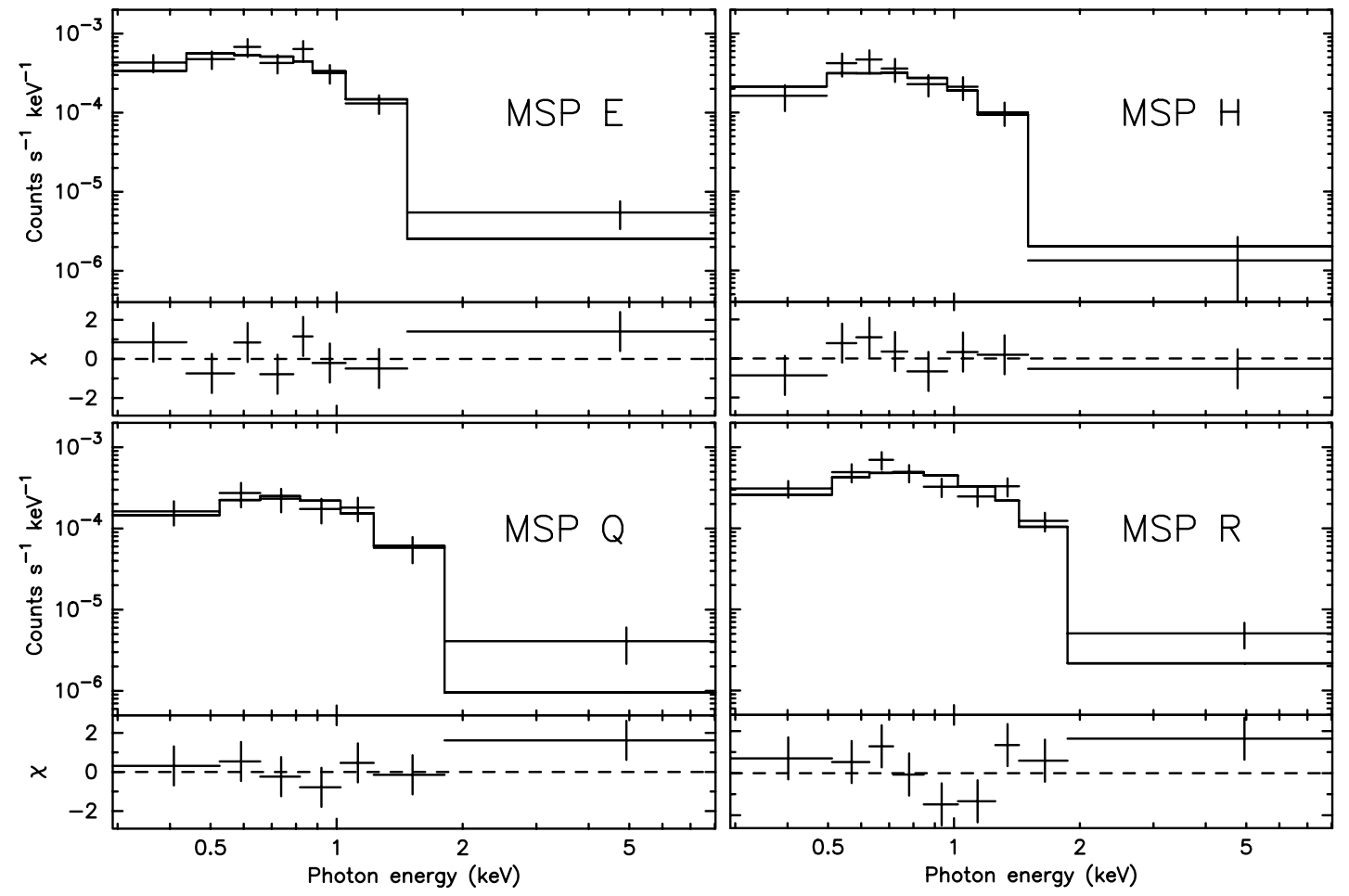

FIG. 3.- Representative X-ray spectra and best-fit single-temperature thermal models (solid line) for the 47 Tuc MSPs. The lower panel for each MSP shows the bestfit residuals. [See the electronic edition of the Journal for spectra of all 47 Tuc MSPs.] 


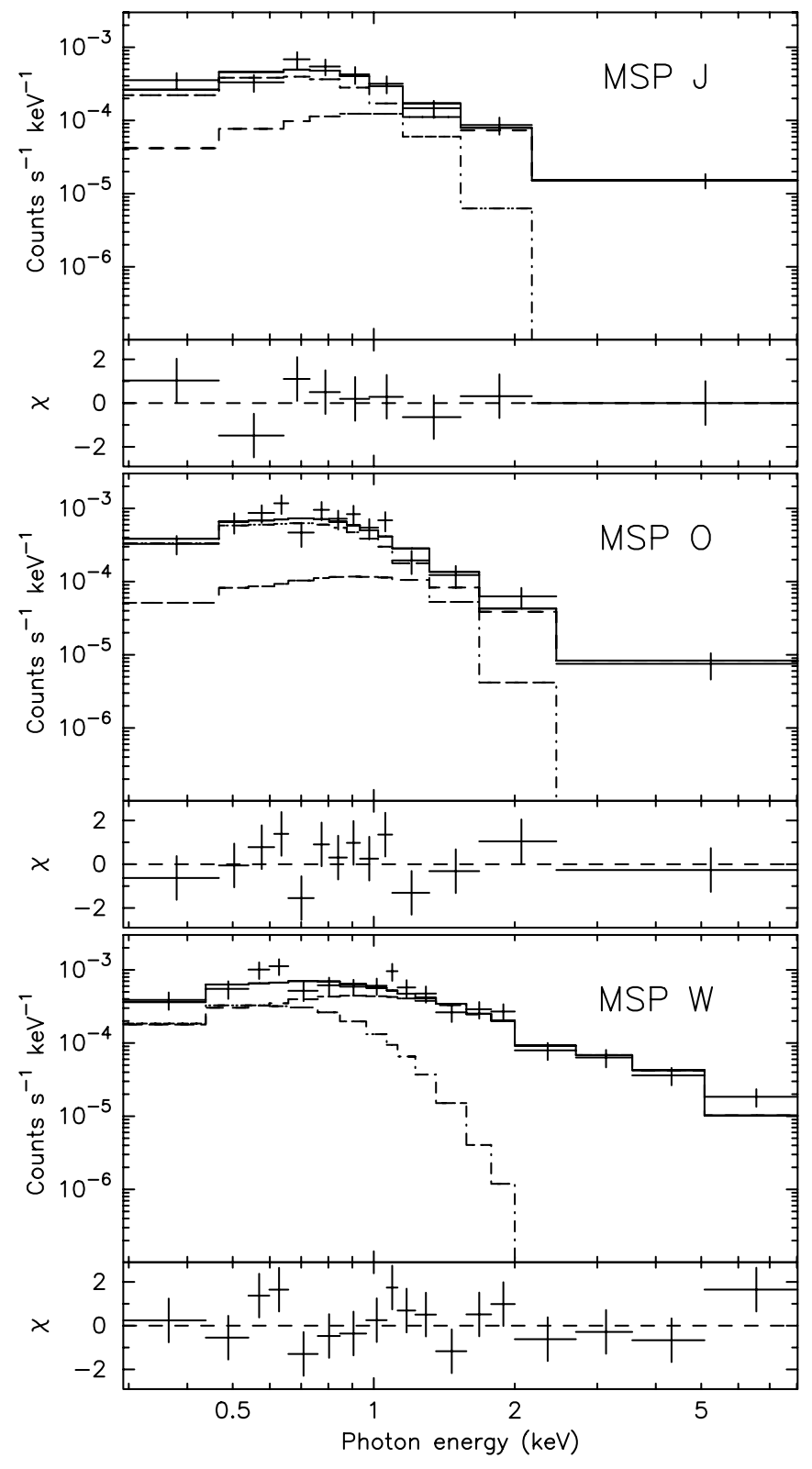

FIG. 4.-X-ray spectra of 47 Tuc J, O, and W, which exhibit nonthermal emission. The solid line shows the best-fit model consisting of a power law (dashed line) and a thermal (dot-dashed line) component. The lower panel for each MSP shows the best-fit residuals.

For the fainter MSPs, such as 47 Tuc C, M, N, Q, and T, a pure power-law model, with a fairly steep spectrum $(\Gamma \gtrsim 2.5)$, also yields an acceptable fit. However, we believe that this likely stems from the fact that a two-temperature spectrum appears power-law-like in the energy range of interest (see, e.g., Fig. 2 in Zavlin et al. 2002). We also note that a broken power-law model, with a break at $E_{b} \approx 1.0 \mathrm{keV}$, is also a good description of the observed emission from the 47 Tuc MSPs. In principle, such a spectrum could arise due to a deficit of radiating high-energy particles in the pulsar magnetosphere. A break in the spectrum is observed in young normal pulsars, although at much higher energies (a few GeV, see, e.g., Thompson 1996). However, evidence against a purely nonthermal model comes from optical observations of PSR J0030+0451, an MSP with a qualitatively similar spectrum to those of the 47 Tuc pulsars, which show that such a model grossly overestimates (by a factor of $\sim 500$ ) the optical flux upper limits when extrapolated to lower energies (Koptsevich et al. 2003).
For the most luminous MSPs in the sample, namely, 47 Tuc J, $\mathrm{O}$, and $\mathrm{W}$, a composite NSA + PL or BB + PL model yields an acceptable fit, with the PL component contributing $\sim 70 \%$, $\sim 50 \%$, and $\sim 75 \%$ of the total flux, respectively. The spectral fits, shown in Figure 4, were found to be acceptable for photon indices $\Gamma \sim 1.0-1.4$. We note that although an $F$-test does not indicate that a composite spectrum is statistically preferred over a pure PL (but with a steeper photon index of $\Gamma \sim 1.5-2.0$ ), the fainter thermal component is very likely genuine, given that the inferred values for $T_{\text {eff }}$ and $R_{\text {eff }}$ are very similar to those of the other 47 Tuc MSPs. The nonthermal X-rays from MSP W, and probably MSP J and $\mathrm{O}$ as well, most likely originate in an intrabinary shock (see $\S 4$ ), although for $\mathrm{J}$ and $\mathrm{O}$ at least a portion of this emission may originate in the pulsar magnetosphere. For $47 \mathrm{Tuc} \mathrm{O}$, which is near the crowded center of the globular cluster, the PL component may also be due to a background X-ray source. The spectra of all other 47 Tuc MSPs may, in fact, contain a faint magnetospheric emission component, which is undetectable in our observations due to the limited number of counts.

It is important to note that in order to model the NSA and BB spectra of MSPs properly, one needs to take into account the rotation of the NS, which induces a modulation in the projected area of the polar cap(s) at the spin period and, in the case of a $\mathrm{NSA}$, in the observed spectrum as well due to the energy dependence of the limb-darkening effects of a NS atmosphere (see, e.g., Romani 1987; Zavlin et al. 1996; Lloyd 2003). Therefore, the derived temperatures and radii represent time-averaged values integrated over the rotation period of the pulsar. As a consequence, the spectral fits may underestimate the true flux by up to a factor of $\sim 2$, even if we consider the effects of gravitational redshift and bending of light near the NS surface (see, e.g., Misner et al. 1970; Pechenick et al. 1983; Beloborodov 2002). Unfortunately, in order to account for these effects, one requires prior knowledge of the relative orientation of the pulsar spin and magnetic axes and the line of sight, which is unavailable.

\section{VARIABILITY ANALYSIS}

Although the limited time resolution of the ACIS-S observations $(3.2 \mathrm{~s})$ precludes a timing analysis at the millisecond level, these observations in conjunction with the 2000 ACIS-I observations permit us to investigate the temporal behavior of the MSP emission over a large range of timescales from hours to years.

As expected, for the bulk of the sample we find no statistically significant X-ray variability. For MSP O, however, there seems to be a gradual decrease in the photon count rate, by a factor of $\sim 2$ in total, especially at medium energies $(0.6-1.5 \mathrm{keV})$, from the second to the fourth ACIS-S observation. There is no known physical mechanism that could account for such a substantial variation of the flux on timescales of days from an old MSP such as 47 Tuc O. On the other hand, such behavior is typical of cataclysmic variables and chromospherically active binaries with $L_{\mathrm{X}} \sim 10^{30}-10^{31} \mathrm{ergs} \mathrm{s}^{-1}$. Thus, we conclude that this variation is most likely due to blending of MSP O with such an X-ray source. This seems likely, as $\mathrm{O}$ is at the heart of the cluster (see Fig. 1), where the number density of X-ray sources is large. MSP R is found to be marginally variable between observations, while MSP U exhibits possible variability within the third observation (Heinke et al. 2005). In both cases, the apparent variability is likely spurious, considering the probability of spurious detection and the number (19) of trials.

For the 12 binary MSPs, we can also examine whether there exist any variations in the X-ray flux as a function of orbital position. In this sense, 47 Tuc J, O, R, and $\mathrm{W}$ are of particular 
interest, as they are in very compact binaries with periods of $2.9,3.3,1.6$, and $3.2 \mathrm{hr}$, respectively. The former three MSPs are bound to very low mass degenerate companions $\left(m_{c} \sim 0.03 M_{\odot}\right)$ and are eclipsed regularly at radio wavelengths for $10 \%-25 \%$ of their orbits (Camilo et al. 2000; Freire 2005). MSP W, on the other hand, has a significantly more massive $\left(m_{c} \gtrsim 0.13 M_{\odot}\right)$ main-sequence secondary (Edmonds et al. 2002) and undergoes eclipses for about $35 \%-40 \%$ of the entire orbit (Freire 2005). For this analysis, the observations for the binary MSPs were first folded at the binary period using the latest values for the orbital periods $P_{b}$ and the epochs of ascending node $T_{0}$ (Freire et al. 2003; P. C. Freire et al. 2006, unpublished data). The net counts were then grouped in phase bins sized so as to allow detection of any large-amplitude variations in the X-ray photon count rate at the eclipse phases. For the same purpose, we also performed Kolmogorov-Smirnov and Cramer-von Mises tests on the folded but unbinned data, using an integer number of binary orbits.

For 47 Tuc J, O, and R we find no detectable variation in the $\mathrm{X}$-ray count rate at any orbital phase and are only able to set very crude upper limits on the amplitude of variation of $\lesssim 60 \%-80 \%$. In contrast, 47 Tuc $\mathrm{W}$ exhibits dramatic variations in the X-ray flux (significant at the $99.9 \%$ level) as a function of orbital phase. This behavior can be naturally explained by the existence of a swept-back shocked stream of gas formed by interaction of the pulsar wind with matter from the irradiated companion issuing through the inner Lagrange point. The properties of this intriguing system and its possible connection to low-mass X-ray binaries are described in depth by Bogdanov et al. (2005).

\section{X-RAY LUMINOSITY VERSUS PULSAR PARAMETERS}

\subsection{X-Ray versus Spin-down Luminosity}

Rotation-powered pulsars, including MSPs, appear to exhibit a linear relation between their X-ray luminosity $L_{X}$ and rotational spin-down luminosity $\dot{E}=4 \pi^{2} I \dot{P}_{i} / P^{3}$, where $I$ is the NS moment of inertia typically assumed to be $10^{45} \mathrm{~g} \mathrm{~cm}^{2}$, with $L_{\mathrm{X}} \sim 10^{-3} \dot{E}$ (Becker \& Trümper 1997; Possenti et al. 2002). However, Grindlay et al. (2002) have shown that the dependence of $L_{\mathrm{X}}$ on $\dot{E}$ for the 47 Tuc MSPs may be significantly flatter, with $L_{\mathrm{X}} \propto \dot{E}^{0.5}$

Using the present data, we can reexamine the $L_{\mathrm{X}}-\dot{E}$ relation for the 47 Tuc MSPs. However, the measured values of $\dot{P}$ and hence $\dot{E}$ for these MSPs are significantly affected by acceleration in the gravitational potential of the globular cluster. In order to obtain the intrinsic period derivative $\dot{P}_{i}$, we first determined the three-dimensional position of each MSP relative to the cluster center by considering the projected radius and assuming that the observed differences in dispersion measure within the MSP sample are solely due to a spread in distance along the line of sight for an assumed uniform intracluster plasma (Freire et al. 2001b). The cluster acceleration term for each MSP was then computed assuming a King model for the cluster using a central velocity dispersion of $v_{z}(0)=11.6 \mathrm{~km} \mathrm{~s}^{-1}$, core radius $r_{c}=23$.' 1 , and distance $D=4.5 \mathrm{kpc}$ (Meylan \& Mayor 1986; Freire et al. 2001b). Subtracting the acceleration term from the observed $\dot{P}$ yields the intrinsic spin-down rate $\dot{P}_{i}$, which was then used to compute $\dot{E}$. The resulting values and their uncertainties are listed in Table 4. In this calculation we have not included MSP H, which exhibits anomalous variations in $\dot{P}$, and MSP W, which currently has no measured $\dot{P}$.

Figure 5 shows the plot of $L_{\mathrm{X}}$ versus $\dot{E}$ for the sample of MSPs in 47 Tuc based on the values listed in Table 4. The distance used to compute $L_{X}$ for the 47 Tuc MSPs was taken to be
TABLE 4

Derived Spin-down and Bolometric Thermal X-Ray Luminosities FOR THE 47 Tuc MSPs

\begin{tabular}{|c|c|c|}
\hline MSP & $\begin{array}{c}\dot{E}^{\mathrm{a}} \\
\left(\times 10^{34} \operatorname{ergs~s}^{-1}\right)\end{array}$ & $\begin{array}{c}L_{\mathrm{X}}^{\mathrm{b}} \\
\left(\times 10^{30} \mathrm{ergs} \mathrm{s}^{-1}\right)\end{array}$ \\
\hline C.. & $0.05_{-0.05}^{+0.12}$ & $2.6_{-2.4}^{+2.9}$ \\
\hline ........................... & $0.67_{-0.21}^{+0.27}$ & $4.8_{-2.7}^{+2.4}$ \\
\hline . & $3.12_{-0.79}^{+0.79}$ & $8.9_{-7.3}^{+9.8}$ \\
\hline $\mathrm{F}^{\mathrm{c}} \ldots$ & $4.09_{-3.16}^{+3.16}$ & $5.5_{-4.2}^{+6.8}$ \\
\hline 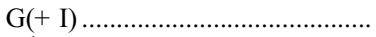 & $<1.72$ & $9.3_{-7.3}^{+9.3}$ \\
\hline . & $\ldots$ & $4.8_{-4.0}^{+5.3}$ \\
\hline .......................... & $<7.14$ & $\ldots$ \\
\hline ........................... & $3.22_{-1.61}^{+1.61}$ & $5.6_{-5.0}^{+4.6}$ \\
\hline (n) & $1.04_{-1.04}^{+1.61}$ & $13.8_{-9.8}^{+12.1}$ \\
\hline 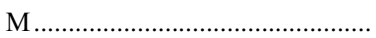 & $\ldots$ & $3.8_{-3.3}^{+5.2}$ \\
\hline $\mathrm{N} \ldots .$. & $1.87_{-1.06}^{+1.49}$ & $3.9_{-3.4}^{+5.5}$ \\
\hline O & $3.12_{-1.13}^{+0.85}$ & $9.3_{-7.6}^{+8.1}$ \\
\hline Q & $1.82_{-0.12}^{+0.12}$ & $3.9_{-3.4}^{+5.5}$ \\
\hline $\mathrm{R}$ & $2.84_{-2.28}^{+3.10}$ & $11.0_{-8.6}^{+12.1}$ \\
\hline $\mathrm{S}^{\mathrm{c}} \ldots \ldots$ & $2.27_{-2.27}^{+0.49}$ & $8.9_{-6.5}^{+9.9}$ \\
\hline ....................... & $1.09_{-0.69}^{+0.41}$ & $2.9_{-2.7}^{+3.1}$ \\
\hline U..... & $3.98_{-0.21}^{+0.21}$ & $5.1_{-4.4}^{+7.8}$ \\
\hline - n........... & -0.21 & $5.9_{-5.9}^{+3.3}$ \\
\hline Y & $4.82_{-4.49}^{+6.12}$ & $3.9_{-3.4}^{+5.5}$ \\
\hline
\end{tabular}

${ }^{\text {a }}$ Values for $\dot{E}$ derived assuming a King model for 47 Tuc using the following parameters: distance $D=4.5 \mathrm{kpc}$, central velocity dispersion $v_{z}(0)=11.6 \mathrm{~km} \mathrm{~s}^{-1}$, core radius $r_{c}=23$.'1, and best-fit constant gas density $n_{e}=0.1 \mathrm{~cm}^{-3}$.

b Values calculated using $L_{\mathrm{X}}=4 \pi R^{2} \sigma_{\mathrm{SB}} T^{4}$, with $R$ and $T$ taken from Table 2, and corrected for gravitational redshift assuming $1+z_{g}=1.31$. For MSP G, $L_{\mathrm{X}}$ is for $\mathrm{G}$ and $\mathrm{I}$ combined.

${ }^{c} L_{\mathrm{X}}$ apportioned based on procedure described in $\S 2$.

d No reliable measurements of $\dot{P}$

$4.5 \mathrm{kpc}$. For the MSP pair F and S, the combined $L_{\mathrm{X}}$ was apportioned based on the procedure described in $\S 2$. In the case of $\mathrm{G}$ and $\mathrm{I}$ the individual contribution of each MSP to the observed $L_{\mathrm{X}}$ is unknown. Also plotted are all MSPs detected in X-rays in the field of the Galaxy as well as in the globular clusters M4, M28, M30, NGC 6397, and NGC 6752. The latest values for the parameters of these MSPs are summarized in Table 5. Here, we have chosen to compute the X-ray luminosity $L_{\mathrm{X}}=4 \pi D^{2} F_{\mathrm{X}}$ in a broad energy band (0.1-10 keV) instead of the ROSAT band $(0.1-2.4 \mathrm{keV})$ in order to take into account the substantial portion of the total flux (up to $\sim 80 \%$ ) that is present beyond $2.4 \mathrm{keV}$ for the MSPs with hard spectra.

In this analysis, we have classified the MSPs based on the three distinct types of X-ray emission: (1) nonthermal magnetospheric, (2) nonthermal shock, and (3) thermal polar cap emission. In the latter category we have also included the MSPs with undetermined spectral properties, as they have very similar luminosities to the pulsars with thermal spectra. In addition, all thermal luminosities have been corrected for the gravitational redshift, assuming $z_{g}=0.31$. For the MSPs with known multicomponent spectra, such as PSR J0437-4715 and 47 Tuc J and O, the emission has been decomposed into the individual constituents.

The thermal luminosities of the 47 Tuc MSPs alone follow the relation $\log L_{X}=(0.24 \pm 1.10) \log \dot{E}+(24 \pm 37)$. In this fit we have taken into account the errors in both $L_{\mathrm{X}}$ and $\dot{E}$ listed in Table 4. As is evident in Figure 5, due to the large uncertainties, the 47 Tuc sample appears consistent with both the linear relation and a much flatter trend, such as $L_{\mathrm{X}} \propto \dot{E}^{0.5}$, predicted by the Harding \& Muslimov (2002) polar cap heating model. It is interesting to note that both field and cluster MSPs with thermal spectra occupy the same region of the $L_{\mathrm{X}}-\dot{E}$ diagram. This 


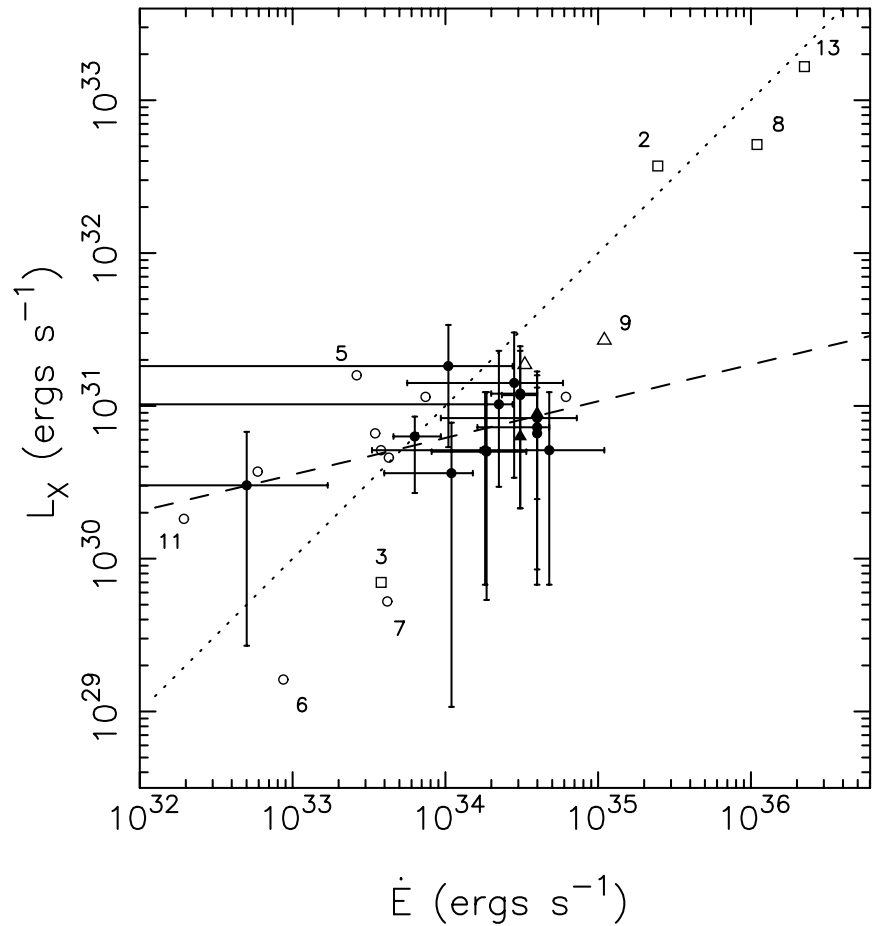

FIG. 5. -X-ray luminosity vs. spin-down luminosity $\left(\dot{E}=4 \pi^{2} I \dot{P}_{i} / P^{3}\right)$ for the MSPs in 47 Tuc ( filled symbols) and all other MSPs detected in X-rays (open symbols). The X-ray luminosities due to polar cap (thermal), magnetospheric, and shock emission are shown as circles, squares, and triangles, respectively. For 47 Tuc $\mathrm{J}$ and $\mathrm{O}$ and J0437-4715 the individual emission components are plotted separately. The dashed line corresponds to the best fit for the 47 Tuc MSPs with thermal spectra, while the dotted line shows the linear relation $L_{\mathrm{X}} \propto$ $10^{-3} \dot{E}$. The numerical labels for the open symbols correspond to the values listed in the first column of Table 5. suggests that in terms of X-ray properties, the two populations are indistinguishable.

Curiously, despite the fundamentally different emission mechanisms, all MSPs appear to follow the linear relation $L_{\mathrm{X}} \propto 10^{-3} \dot{E}$ and have an efficiency of converting $\dot{E}$ to $L_{\mathrm{X}}$ covering a surprisingly narrow range, with $L_{\mathrm{X}} / \dot{E} \sim 10^{-4}$ to $10^{-3}$. This is not expected given that in systems such as 47 Tuc J, O, and W, B1957+20, and $\mathrm{J} 1740-5340$, whose emission most likely originates in an intrabinary shock, $L_{\mathrm{X}}$ is determined, in part, by parameters unrelated to the physics of the pulsar, such as the binary separation, the properties of the companion star, and the volume of the shocked region. The small range in $L_{X} / \dot{E}$ may stem from the fact that in all cases the high-energy emission is, ultimately, driven by the same underlying particle acceleration and pair production processes occurring in the pulsar magnetosphere. However, the fact that MSPs with $\dot{E} \lesssim 10^{35} \mathrm{ergs} \mathrm{s}^{-1}$ appear to have thermal spectra, whereas those with $\dot{E} \sim 10^{36} \mathrm{ergs} \mathrm{s}^{-1}$ show strong nonthermal emission, suggests a profound difference in the conditions in the pulsar magnetosphere (see $\S 6.3$ ).

\subsection{X-Ray Luminosity versus Magnetic Field Strength}

Next, we consider how $L_{\mathrm{X}}$ depends on the pulsar magnetic field as it governs the physical processes responsible for both magnetospheric emission and polar cap heating, and, to some degree, shock emission. In Figure $6 a$, we plot $L_{\mathrm{X}}$ versus $B_{\mathrm{lc}}=$ $9.35 \times 10^{5}\left(P / 10^{-3} \mathrm{~s}\right)^{-5 / 2}\left(\dot{P} / 10^{-20}\right)^{1 / 2} \mathrm{G}$, the magnetic field at the light cylinder $\left(r_{c}=c P / 2 \pi\right)$ for a simple dipole. The MSPs with thermal spectra are consistent with $\log L_{\mathrm{X}}=(0.39 \pm 0.54) \log B_{\mathrm{lc}}+$ (28 \pm 3 ). As with the $L_{X}-\dot{E}$ relation, the fit is not particularly constraining due to the large uncertainties. Nonetheless, as noted by Saito et al. (1997), it is striking that B1821-24 and B1937+21, which exhibit very luminous nonthermal (magnetospheric) X-ray emission, have the highest magnetic fields at the light cylinder, with $B_{\mathrm{lc}}>10^{5.5} \mathrm{G}$. It may be the case that these MSPs do, in

TABLE 5

Parameters for MSPs Detected in X-Rays outside of 47 Tuc

\begin{tabular}{|c|c|c|c|c|c|c|}
\hline Number & $\mathrm{MSP}^{\mathrm{a}}$ & $\begin{array}{c}P \\
(\mathrm{~ms})\end{array}$ & $\begin{array}{c}\dot{P}_{i} \\
\left(10^{-20}\right)\end{array}$ & $\begin{array}{c}D \\
(\mathrm{kpc})\end{array}$ & $\begin{array}{c}F_{\mathrm{X}}(0.1-10 \mathrm{keV})^{\mathrm{b}} \\
\left(10^{-13} \mathrm{ergs}^{-2} \mathrm{sm}^{-1}\right)\end{array}$ & References \\
\hline 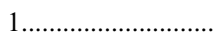 & $\mathrm{J} 0030+0451$ & 4.86 & $<1.0^{\mathrm{c}}$ & 0.3 & 3.6 & 1,2 \\
\hline $2 \ldots \ldots \ldots \ldots \ldots \ldots \ldots$ & $\mathrm{J} 0218+4232$ & 2.32 & 7.78 & 2.7 & 4.2 & 3,4 \\
\hline 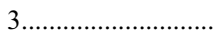 & $\mathrm{J} 0437-4715$ & 5.76 & 1.86 & $0.139^{\mathrm{d}}$ & $12.9(3.0)$ & 5,6 \\
\hline $4 \ldots \ldots \ldots \ldots \ldots \ldots \ldots$ & $\mathrm{J} 0751+1807$ & 3.48 & 0.73 & 1.1 & 0.47 & 7,8 \\
\hline $5 \ldots \ldots \ldots \ldots \ldots \ldots \ldots \ldots$ & $\mathrm{J} 1012+5307$ & 5.26 & 0.97 & $>0.77^{\mathrm{d}}$ & 1.3 & 9,8 \\
\hline 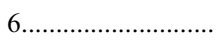 & J1024-0719 & 5.16 & $<0.30^{\mathrm{c}}$ & $<0.20^{\mathrm{d}}$ & 0.2 & $10,11,12$ \\
\hline $7 \ldots \ldots \ldots \ldots \ldots \ldots \ldots$ & $\mathrm{J} 1744-1134$ & 4.07 & 0.71 & $0.357^{\mathrm{d}}$ & 0.2 & $10,13,12$ \\
\hline 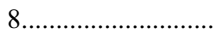 & B1937+21 & 1.56 & 10.6 & 3.6 & 3.3 & 11,14 \\
\hline 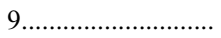 & $\mathrm{B} 1957+20$ & 1.61 & 1.15 & 2.5 & 0.9 & 11,15 \\
\hline 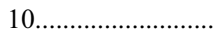 & $\mathrm{J} 2124-3358$ & 4.93 & 1.30 & 0.25 & 3.6 & $10,11,12$ \\
\hline 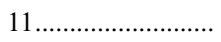 & B1620-26 (M4) & 11.07 & $\sim 0.67^{\mathrm{c}}$ & $1.73^{\mathrm{d}}$ & 0.03 & $16,17,18$ \\
\hline $12 \ldots \ldots \ldots \ldots \ldots \ldots$ & $\mathrm{J} 1740-5340$ ( NGC 6397) & 3.60 & 3.90 & $2.55^{\mathrm{d}}$ & 0.24 & $19,20,21$ \\
\hline $13 \ldots \ldots \ldots \ldots \ldots \ldots \ldots \ldots \ldots$ & B1821-24 (M28) & 3.05 & 162 & $5.5^{\mathrm{d}}$ & 4.6 & 22,23 \\
\hline $14 \ldots \ldots \ldots \ldots \ldots \ldots \ldots \ldots$ & J1911-6000C (NGC 6752) & 5.28 & 0.22 & $4.1^{\mathrm{d}}$ & 0.01 & 24 \\
\hline 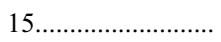 & $\mathrm{J} 2140-2310 \mathrm{~A}(\mathrm{M} 30)$ & 11.02 & $<209^{\mathrm{c}}$ & $9.0^{\mathrm{d}}$ & $\lesssim 0.007$ & 25,26 \\
\hline
\end{tabular}

${ }^{\text {a }}$ For the globular cluster MSPs the host cluster is given in parentheses.

b Total flux. For J0437-4715 the value in parentheses gives the nonthermal flux.

c Poorly determined acceleration corrections.

d Accurate distances/limits. Other estimates are obtained primarily from the dispersion measure together with the Cordes \& Lazio (2002) electron density model and are rather uncertain (up to a factor of $\sim 2$ ).

REFERENCES.- (1) Lommen et al. 2000; (2) Becker \& Aschenbach 2002; (3) Navarro et al. 1995; (4) Webb et al. 2004a; (5) van Straten et al. 2001; (6) Zavlin et al. 2002; (7) Lundgren et al. 1995; (8) Webb et al. 2004b; (9) Lange et al. 2001; (10) Toscano et al. 1998; (11) Toscano et al. 1999b; (12) Becker \& Trümper 1999; (13) Toscano et al. 1999a; (14) Cusumano et al. 2003; (15) Stappers et al. 2003; (16) Lyne et al. 1988; (17) Richer et al. 1997; (18) Bassa et al. 2004; (19) D’Amico et al. 2001; (20) Bassa \& Stappers 2004; (21) Grindlay et al. 2002; (22) Cognard \& Backer 2004; (23) Becker et al. 2003; (24) D’Amico et al. 2002; (25) Carretta et al. 2000; (26) Ransom et al. 2004. 


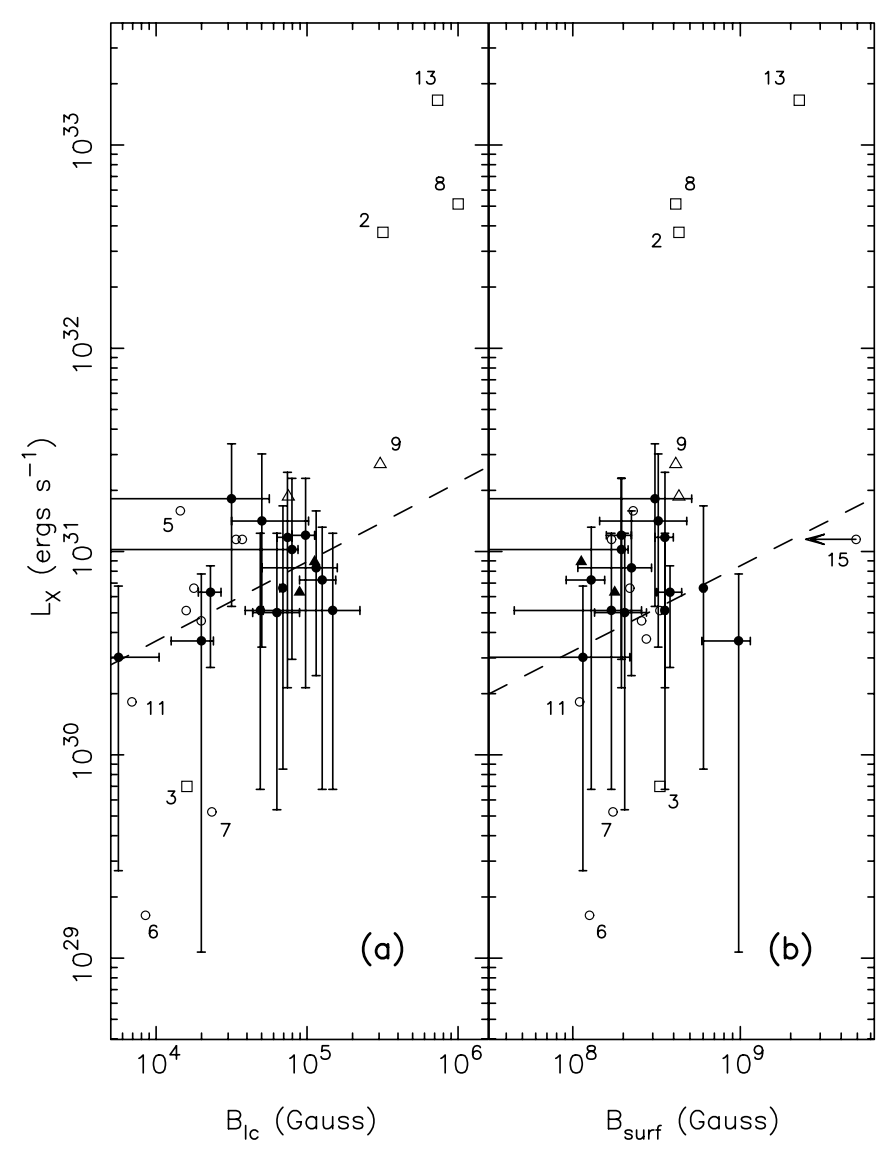

FIG. 6.-(a) $L_{\mathrm{X}}$ vs. magnetic field strength at the light cylinder $\left(B_{\mathrm{lc}}\right)$ for the MSPs in 47 Tuc (filled symbols) and all other MSPs detected in X-rays (open symbols). (b) $L_{\mathrm{X}}$ vs. surface magnetic field strength $\left(B_{\text {surf }}\right)$. The dashed line corresponds to the best fit for the 47 Tuc MSPs with thermal spectra. The various symbols are defined as in Fig. 5.

fact, have heated polar caps, but the resulting emission is several orders of magnitude fainter than the magnetospheric emission. Alternatively, the energetics of the system may not favor substantial polar cap heating (see, e.g., Harding \& Muslimov 2002). Pulsars with $B_{\mathrm{lc}}<10^{5} \mathrm{G}$, on the other hand, appear to have X-ray emission that is typically either thermal or of indeterminate (but probably thermal) character.

In the case of the dipole magnetic field at the surface $B_{\text {surf }}=$ $3.2 \times 10^{19}(P \dot{P})^{1 / 2} \mathrm{G}$, the $L_{\mathrm{X}}-B_{\text {surf }}$ relation (Fig. $6 b$ ) seems even less defined, with a large scatter for both the complete MSP sample and the 47 Tuc MSPs. The thermal $L_{X}$ for the 47 Tuc MSPs is best fit by $\log L_{\mathrm{X}}=(0.36 \pm 1.27) B_{\text {surf }}+(22 \pm 37)$. The apparent lack of correlation between $L_{\mathrm{X}}$ and $B_{\text {surf }}$ may indicate either that the small-scale magnetic field structure near the surface of the polar caps deviates from that of a pure dipole (see, e.g., Zhang \& Cheng 2003) or that both the thermal and nonthermal X-ray emission properties of MSPs are determined by the conditions much higher in the magnetosphere (e.g., at the light cylinder).

\subsection{Uncertainties in X-Ray Luminosity and Pulsar Parameters}

In comparing theoretical predictions and observed relations, it is necessary to examine and quantify all the effects that act to modify the observed parameters under consideration. First, it is important to emphasize that the X-ray luminosities plotted in Figures 5 and 6 represent observed, not true quantities. This is especially critical for the nonthermal magnetospheric emission, which is subject to beaming and hence viewing angle effects. Therefore, the measured value of $L_{X}$ may greatly underestimate the true X-ray luminosity. As for the thermal emission, a major source of uncertainty is introduced by the lack of knowledge of the masses and radii of the sample of MSPs. These parameters affect $L_{X}$ through the gravitational redshift and bending of light. In particular, the true thermal luminosity, as measured at the NS surface, is larger by a factor of $\left(1+z_{g}\right)^{2}$ than the luminosity measured by a distant observer. In our analysis, we have corrected for this effect by assuming the canonical values of $M=$ $1.4 M_{\odot}$ and $R=10 \mathrm{~km}$. However, for a reasonable range of NS masses, 1.2-2.3 $M_{\odot}$, based on the results of Lyne et al. (2004) and Nice et al. (2005), we find that the true $L_{\mathrm{X}}$ may differ from the value used here by up to a factor of $\sim 1.8$. In addition, for NS radii in the range $9-16 \mathrm{~km}$, the derived $L_{\mathrm{X}}$ may deviate from the true value by $\lesssim 20 \%$.

The parameters $\dot{E}, B_{\text {surf }}$, and $B_{\mathrm{lc}}$ also depend on the MSP mass and radius, as $\dot{E} \propto M R^{2}$ and $B \propto\left(M R^{2}\right)^{1 / 2} / R^{3}$. For the same range of NS masses $\left(1.2-2.3 M_{\odot}\right)$, the assumption of $1.4 M_{\odot}$ may result in a deviation of up to $\sim 60 \%$ for $\dot{E}$ and $\sim 25 \%$ for $B_{\text {surf }}$ and $B_{\text {lc }}$ from the true values. This implies that a spread in masses within the sample of MSPs would tend to increase the scatter about the derived relations in both coordinates (e.g., $L_{\mathrm{X}}$ and $\dot{E}$ ) and perhaps result in a skewed trend. Conversely, for a sample of MSPs that have very similar masses but are systematically more or less massive than the canonical $1.4 M_{\odot}$, the result will be an offset in the derived intercept, with little effect on the slope of the $\log$-log relation under consideration (e.g., $\log L_{\mathrm{X}}-\log \dot{E}$ relation). As for $R_{\mathrm{NS}}$, for the range $9-16 \mathrm{~km}$ we find that $\dot{E}, B_{\text {surf }}$, and $B_{\mathrm{lc}}$ may differ by up to a factor of $\sim 2.6$. The effect of the NS radius on the scatter in the trends discussed above is uncertain due to a lack of knowledge of the true NS equation of state (EOS). For instance, in certain theoretical models of the NS EOS a small change in $M_{\mathrm{NS}}$ does not result in an appreciable change in $R_{\mathrm{NS}}$, while for others the opposite is the case (see, e.g., Lattimer \& Prakash 2001 for details).

For the 47 Tuc MSPs, the parameters derived from $\dot{P}$ are subject to an additional uncertainty arising from a lack of knowledge of the magnitude of acceleration of the MSPs by the gravitational potential of the globular cluster. It is important to note that the model-dependent values derived for $\dot{P}_{i}$ are sensitive to the assumptions made regarding the gravitational potential and gas density profile of the cluster. For simplicity, in this paper we have derived $\dot{P}_{i}$ for each MSP assuming a simple constant gas density profile. However, it is likely that in reality the gas distribution of 47 Tuc is more complex. For instance, the nearly identical dispersion measures of the MSPs near the cluster center, namely, 47 Tuc F, L, O, and S (Freire et al. 2003), may be interpreted as being due to the presence of a cavity in the plasma around the cluster core carved out by the winds of these pulsars. Hence, obtaining accurate values of $\dot{P}_{i}$ may require implementation of more elaborate models of the gas distribution, which will be explored in detail elsewhere.

Another potential source of systematic uncertainty is the presence of an observational selection effect. It is probable that so far pulsar observations have sampled only the most luminous and nearby MSPs. The existence of X-ray-faint MSPs, both in globular clusters and the field of the Galaxy, would mean that the observed narrow range of $L_{X}$ for the MSPs with thermal spectra is simply an artifact of the limited sample of very low luminosity MSPs.

Given the large uncertainties discussed above, any conclusions drawn for the apparent relations between $L_{X}$ and quantities derived from the pulsar spin parameters should be taken with much caution, as it is not possible, at present, to properly account for all effects that act to modify the observed trends. 


\section{DISCUSSION}

\subsection{X-Ray Properties of MSPs}

The spectral fits described in $\oint 3$ show that the majority of 47 Tuc MSPs exhibit emission consistent with a thermal model, with no indication of any magnetospheric emission. It is probable that the MSPs with seemingly purely thermal emission do, in fact, have a fainter magnetospheric X-ray component $\left(<10^{30} \mathrm{ergs} \mathrm{s}^{-1}\right)$. However, it remains unclear what determines the relative strengths of these two X-ray production mechanisms. Although viewing angle dependences and beaming of the magnetospheric radiation can explain why thermal emission dominates in many MSPs, it is more likely that the relative strength of the two components is determined by the poorly understood details of the magnetospheric emission and polar cap heating mechanisms.

Grindlay et al. (2002) and Cheng \& Taam (2003) have raised the possibility that the very narrow range of thermal luminosities and small emission areas imply the existence of a strong multipole magnetic field near the surface. However, the small range in $L_{\mathrm{X}}$ is consistent with a simple dipole field if one considers general relativistic effects near the surface. In particular, gravitational bending of light causes $\gtrsim 75 \%$ of the NS surface to be visible at any given time (see, e.g., Pechenick et al. 1983; Pavlov \& Zavlin 1997; Beloborodov 2002). As a consequence, the degree of modulation in the flux that arises due to rotation of the NS is greatly reduced. This, in turn, diminishes the scatter in $L_{\mathrm{X}}$ for the sample of MSPs that would otherwise result from different orientations of the magnetic and spin axes relative to the line of sight. Thus, although entirely plausible, the existence of a multipole magnetic field need not be invoked to account for the small range in $L_{\mathrm{X}}$.

We note that the inferred radii of the thermally emitting areas obtained in $\S 3$ are indeed somewhat smaller than the expected size of a magnetic polar cap for a simple dipole field, $R_{\mathrm{pc}}=$ $\left(2 \pi R_{\mathrm{NS}} / c P\right)^{1 / 2} R_{\mathrm{NS}}$ (e.g., $R_{\mathrm{pc}}=2.6 \mathrm{~km}$ for a $3 \mathrm{~ms}$ pulsar). This likely arises due to the fact that we have fitted most MSP spectra with a single-temperature model, where in reality a two-temperature model is more appropriate, as suggested by the results of $\S 3$. In addition, due to variation of the projected area of the polar caps, induced by the rotation of the star, fits to the time-integrated spectrum result in an underestimate of the effective emission area. However, even with a two-temperature spectrum and a correction factor for the effective area, in the case of a BB model the total emitting region is still substantially smaller than the expected polar cap area. If the NS surface does, in fact, radiate like a blackbody, this discrepancy may be indicative of either nonuniform polar cap heating or deviations from a pure dipole field. For instance, the polar cap model of Harding \& Muslimov (2002) predicts that the heating rate by positrons should be highest near the rim of the polar cap (cf. Fig. 7 of Harding \& Muslimov 2002), resulting in an annulus-shaped emitting region with an area significantly smaller than that of the entire polar cap. Chen et al. (1998) have argued that a small emission area can arise if the MSP is an aligned rotator, resulting in a so-called squeezed polar cap. The outer gap model of Zhang \& Cheng (2003) also predicts that the heated area should be significantly smaller than the whole polar cap due to the presence of a small-scale multipole field near the surface. Finally, this result may imply that a blackbody is simply not a valid approximation of the NS surface. The discrepancy may thus be a consequence of the fact that simplified blackbody models result in higher derived temperatures and smaller emission areas due to the fact that the X-ray spectra of light-element atmospheres are harder than blackbody spectra (Zavlin et al. 1996).
The results of this study as well as recent Chandra and XMMNewton observations of nearby field MSPs (Zavlin et al. 2002; Becker \& Aschenbach 2002; Webb et al. 2004a, 2004b) have revealed that there are no clear systematic differences between the X-ray properties of globular cluster and field MSPs, which could arise, for instance, due to different evolutionary paths. For example, the nearby field MSPs, such as J0437-4715 and J0030+ 0451, are quite similar to the bulk of 47 Tuc MSPs in terms of $\mathrm{X}$-ray luminosity and spectral properties. In addition, B1821-24 in the globular cluster M28 is akin to B1937+21, characterized by large $X$-ray and spin-down luminosities $\left(L_{\mathrm{X}}>10^{33} \mathrm{ergs} \mathrm{s}^{-1}\right.$, $\dot{E}>10^{36}$ ergs s$\left.^{-1}\right)$, hard nonthermal spectra, and very narrow radio and X-ray pulse profiles (Becker \& Trümper 1999). These similarities imply that if, in fact, multiple binary exchanges and accretion episodes occur for globular cluster MSPs, they may not significantly alter the elementary emission properties of the pulsar.

\subsection{The Eclipsing Binary MSPs}

Bogdanov et al. (2005) have shown that the binary MSP 47 Tuc W exhibits large-amplitude X-ray variability at the binary period. This modulation can be interpreted as being due to geometric occultations of an X-ray-emitting intrabinary shock by the $\sim 0.15 M_{\odot}$ main-sequence companion. On the other hand, in the three other systems in which radio eclipses are observed, namely, 47 Tuc J, O, and R, we detect no such variability. The persistence of X-ray emission during the radio eclipses confirms that at radio frequencies $\mathrm{J}, \mathrm{O}$, and $\mathrm{R}$ are not eclipsed by their respective binary companions but rather by a tenuous ionized envelope formed due to mass loss by the companion driven by the pulsar wind, as in the case of PSRs B1957+20 and J2051-0827 (Arzoumanian et al. 1994; Stappers et al. 1996).

The unique X-ray behavior of 47 Tuc $\mathrm{W}$ is most certainly the result of the presence of a main-sequence secondary star instead of the typical very low mass $\left(\sim 0.03 M_{\odot}\right)$ degenerate companion found in "black widow"-like systems such as J, O, and R. While the latter three systems are expected end products of low-mass $\mathrm{X}$-ray binary evolution, 47 Tuc W is likely the result of an exchange encounter in which the original very low-mass companion was ejected from the binary (Freire 2005 and references therein). However, even if the current companion of MSP W is Roche lobe filling, the existence of an intrabinary shock implies that another accretion phase cannot commence, as the energetic pulsar wind is constraining the outflow of mass from the companion (see Bogdanov et al. 2005 for details).

\subsection{X-Ray versus Radio MSP Properties}

In $\oint 5$ we have found that the $\log L_{X}-\log \dot{E}$ relation for the thermally emitting MSPs has a slope of $\beta \sim 0.2 \pm 1.1$. Although such a trend is consistent with the prediction of the polar cap heating model of Harding \& Muslimov (2002), $L_{\mathrm{X}} \propto \dot{E}^{0.5}$, we cannot exclude the possibility that the 47 Tuc MSPs follow the linear $L_{\mathrm{X}}-\dot{E}$ relation.

Despite the large uncertainties, for the present sample of MSPs detected in X-rays we still observe a general trend of decrease of $L_{\mathrm{X}}$ as a function of spin-down luminosity and magnetic field strength. If these parameters are, in turn, determined by the age of the MSP, it is tempting to speculate that Figures 5 and 6 represent evolutionary diagrams. Here we consider a possible evolutionary path of a MSP described within the framework of the Harding \& Muslimov (2002) electron-positron $\left(e^{ \pm}\right)$pair production model. For relatively young and energetic MSPs, such as PSR B1821-24 $\left(\tau \sim 3 \times 10^{7} \mathrm{yr}\right), e^{ \pm}$pairs are produced via 
curvature radiation (CR). These pairs, in turn, produce the observable $\mathrm{X}$-ray $/ \gamma$-ray and radio nonthermal magnetospheric emission. Although some polar cap heating by a backflow of energetic particles may occur in these objects, the nonthermal component $\left(\sim 10^{33} \mathrm{ergs} \mathrm{s}^{-1}\right)$ is several orders of magnitude brighter, making it difficult to observe any emission from the polar caps. As the MSP ages, it undergoes rotational spin-down and perhaps magnetic field decay, which result in a decline in the CR pair production rate. At a certain stage, the magnetic field becomes too low to permit $\mathrm{CR}$ pair production, so inverse Compton scattering (ICS) of soft X-rays by accelerated electrons becomes an important channel for $e^{ \pm}$pair production. ICS pair production results in substantial polar cap heating by a backflow of positrons from the magnetosphere. Since the polar cap heating rate has a weaker dependence on the spin-down luminosity $\left(L_{h} \propto \dot{E}^{1 / 2}\right)$ than magnetospheric emission $\left(L_{m} \propto \dot{E}\right)$, the thermal emission will eventually become dominant over the magnetospheric emission, as is observed in the majority of 47 Tuc MSPs and many field MSPs. At much later times, the MSP is expected to cross the ICS pair production death line (see Fig. 1 in Harding et al. 2002) and will no longer be observable as a pulsar, both in the radio and $\mathrm{X}$-rays. In the end, as the polar caps cool, the MSP will fade away from view at X-ray energies as well.

\section{CONCLUSION}

We have presented a spectral analysis of the X-ray emission for the complete sample of known MSPs in 47 Tuc. The X-ray spectra of the majority of MSPs are well described by a thermal model, a BB, or a NS hydrogen atmosphere, with $T_{\text {eff }} \sim$ $(1-3) \times 10^{6} \mathrm{~K}, R_{\mathrm{eff}} \sim 0.1-3 \mathrm{~km}$, and $L_{\mathrm{X}} \sim 10^{30}-10^{31} \mathrm{ergs} \mathrm{s}^{-1}$ $(0.3-8 \mathrm{keV})$. The small observed scatter in the thermal $L_{\mathrm{X}}$ is consistent with a simple dipole magnetic field configuration. A two-temperature thermal model, reminiscent of that observed from some nearby field MSPs, is also consistent with the emission from the bulk of 47 Tuc MSPs and, in some cases, results in an improved fit. Such a spectrum could arise due to nonuniform polar cap heating or lateral diffusion of heat across the NS surface away from the regions directly heated by particles from the magnetosphere. The MSPs J, O, and $\mathrm{W}$ show a prominent nonthermal component in their spectra. At least some of the nonthermal X-rays are very likely due to interaction of the relativistic particle wind of the pulsar with matter from the companion. All other 47 Tuc MSPs exhibit no evidence of a significant nonthermal $\mathrm{X}$-ray component.

Our reexamination of the $L_{X}-\dot{E}$ relation has revealed that regardless of the emission mechanism responsible for production of the observed X-rays, the conversion efficiency of $\dot{E}$ to $L_{\mathrm{X}}$ appears to be, in all cases, $\sim 10^{-4}$ to $10^{-3}$.

All of the 47 Tuc MSPs, with the exception of 47 Tuc W, display no large-amplitude variability in their X-ray emission on timescales of days, weeks, and years. The MSP W exhibits dramatic variations at the binary period, which can be attributed to the presence of an intrabinary shock that is eclipsed by the secondary star. The lack of long-term variations in the flux of most MSPs could potentially serve as a criterion for classification of unidentified soft X-ray sources (Heinke et al. 2005).

Given that most of the $\sim 100$ MSPs currently known have spin parameters similar to those of the 47 Tuc MSPs, we expect many of them to have predominantly thermal X-ray spectra. Moreover, for these MSPs, gravitational bending of light near the NS surface ensures that at least one of the antipodal heated polar caps is always observable, while for a wide range of angles between the magnetic and spin axes and the line of sight both polar caps are visible simultaneously for a large portion of the MSP spin period (see, e.g., Nollert et al. 1989). This effect invariably results in a $100 \%$ duty cycle, even if the magnetospheric emission beams never sweep across the Earth or if the radio emission is very faint or heavily absorbed/scattered. Therefore, many MSPs with heated polar caps may, in principle, be detectable at $\mathrm{X}$-ray energies even if they cannot be observed as radio pulsars.

Finally, based on our results, we conclude that in terms of the characteristics of their X-ray emission, there is no clear separation between MSPs in the field of the Galaxy and in globular clusters.

We would like to thank Peter Edmonds for insightful discussions, Don Lloyd for use of his NS hydrogen atmosphere model, and the referee Natalie Webb for many helpful comments. This work was supported by NASA Chandra grants GO2-3059A and G02-3059B. The research presented here has made use of the NASA Astrophysics Data System.

Facilities: CXO
Arzoumanian, Z., Fruchter, A. S., \& Taylor, J. H. 1994, ApJ, 426, L85

Backer, D. C., Kulkarni, S. R., Heiles, C., Davis, M. M., \& Goss, W. M. 1982, Nature, 300, 615

Bassa, C. G., \& Stappers, B. W. 2004, A\&A, 425, 1143

Bassa, C. G., et al. 2004, ApJ, 609, 755

Becker, W., \& Aschenbach, B. 2002, in Neutron Stars, Pulsars, and Supernova Remnants, ed. W. Becker, H. Lech, \& J. Trümper (MPE Rep. 278; Garching: MPE), 64

Becker, W., \& Trümper, J. 1997, A\&A, 326, 682

- 1999, A\&A, 341, 803

Becker, W., et al. 2003, ApJ, 594, 798

Beloborodov, A. M. 2002, ApJ, 566, L85

Bogdanov, S., Grindlay, J. E., \& van den Berg, M. 2005, ApJ, 630, 1029

Camilo, F., Lorimer, D. R., Freire, P., Lyne, A. G., \& Manchester, R. N. 2000, ApJ, 535, 975

Camilo, F., \& Rasio, F. A. 2005, in ASP Conf. Ser. 328, Binary Radio Pulsars, ed. F. A. Rasio \& I. H. Stairs (San Francisco: ASP), 147

Cardelli, J. A., Clayton, G. C., \& Mathis, J. S. 1989, ApJ, 345, 245

Carretta, E., Gratton, R. G., Clementini, G., \& Fusi Pecci, F. 2000, ApJ, 533, 215

Chen, K., Ruderman, M., \& Zhu, T. 1998, ApJ, 493, 397

Cheng, K. S., \& Taam, R. E. 2003, ApJ, 598, 1207

Cognard, I., \& Backer, D. C. 2004, ApJ, 612, L125

Cordes, J. M., \& Lazio, T. J. W. 2002, preprint (astro-ph/0207156)

Cusumano, G., et al. 2003, A\&A, 410, L9

\section{REFERENCES}

D’Amico, N., Lyne, A. G., Manchester, R. N., Possenti, A., \& Camilo, F. 2001, ApJ, 548, L171

D’Amico, N., Possenti, A., Fici, L., Manchester, R. N., Lyne, A. G., Camilo, F., \& Sarkissian, J. 2002, ApJ, 570, L89

Edmonds, P. D., Gilliland, R. L., Camilo, F., Heinke, C. O., \& Grindlay, J. E. 2002, ApJ, 579, 741

Freire, P. C. 2005, in ASP Conf. Ser. 328, Binary Radio Pulsars, ed. F. A. Rasio \& I. H. Stairs (San Francisco: ASP), 405

Freire, P. C., Camilo, F., Kramer, M., Lorimer, D. R., Lyne, A. G., Manchester, R. N., \& D' Amico, N. 2003, MNRAS, 340, 1359

Freire, P. C., Camilo, F., Lorimer, D. R., Lyne, A. G., Manchester, R. N., \& D'Amico, N. 2001a, MNRAS, 326, 901

Freire, P. C., Kramer, M., Lyne, A. G., Camilo, F., Manchester, R. N., \& D’Amico, N. 2001b, ApJ, 557, L105

Gratton, R. G., Bragaglia, A., Carretta, E., Clementini, G., Desidera, S., Grundahl, F., \& Lucatello, S. 2003, A\&A, 408, 529

Grindlay, J. E., Camilo, F., Heinke, C. O., Edmonds, P. D., Cohn, H., \& Lugger, P. 2002, ApJ, 581, 470

Grindlay, J. E., Heinke, C., Edmonds, P. D., \& Murray, S. S. 2001, Science, 292, 2290

Harding, A. K., \& Muslimov, A. G. 2002, ApJ, 568, 862

Harding, A. K., Muslimov, A. G., \& Zhang, B. 2002, ApJ, 576, 366

Heinke, C. O., Grindlay, J. E., Edmonds, P. D., Cohn, H. N., Lugger, P. M., Camilo, F., Bogdanov, S., \& Freire, P. C. 2005, ApJ, 625, 796 
Koptsevich, A. B., Lundqvist, P., Serafimovich, N. I., Shibanov, Yu. A., \& Sollerman, J. 2003, A\&A, 400, 265

Lange, C., Camilo, F., Wex, N., Kramer, M., Backer, D. C., Lyne, A. G., \& Doroshenko, O. 2001, MNRAS, 326, 274

Lattimer, J. M., \& Prakash, M. 2001, ApJ, 550, 426

Lloyd, D. A. 2003, MNRAS, submitted (astro-ph/0303561)

Lommen, A. N., Zepka, A., Backer, D. C., McLaughlin, M., Cordes, J. M., Arzoumanian, Z., \& Xilouris, K. 2000, ApJ, 545, 1007

Lundgren, S. C., Zepka, A. F., \& Cordes, J. M. 1995, ApJ, 453, 419

Lyne, A. G., Biggs, J. D., Brinklow, A., McKenna, J., \& Ashworth, M. 1988, Nature, 332, 45

Lyne, A. G., et al. 2004, Science, 303, 1153

Manchester, R. N., Lyne, A. G., Johnston, S., D’Amico, N., Lim, J., \& Kniffen, D. A. 1990 , Nature, 345,598

Manchester, R. N., Lyne, A. G., Robinson, C., Bailes, M., \& D'Amico, N. 1991, Nature, 352, 219

Meylan, G., \& Mayor, M. 1986, A\&A, 166, 122

Misner, C. W., Thorne, K. S., \& Wheeler, J. A. 1970, Gravitation (New York: Freeman)

Navarro, J., de Bruyn, G., Frail, D., Kulkarni, S. R., \& Lyne, A. G. 1995, ApJ, 455, L55

Nice, D. J., Splaver, E. M., Stairs, I. H., Löhmer, O., Jessner, A., Kramer, M., \& Cordes, J. M. 2005, ApJ, 634, 1242

Nollert, H.-P., Ruder, H., Herold, H., \& Kraus, U. 1989, A\&A, 208, 153

Pavlov, G. G., \& Zavlin, V. E. 1997, ApJ, 490, L91

Pechenick, K. R., Ftaclas, C., \& Cohen, J. M. 1983, ApJ, 274, 846

Possenti, A., Cerutti, R., Colpi, M., \& Mereghetti, S. 2002, A\&A, 387, 993

Predehl, P., \& Schmitt, J. H. M. M. 1995, A\&A, 293, 889
Ransom, S. M., Stairs, I. H., Backer, D. C., Greenhill, L. J., Hessels, J. W. T., \& Kaspi, V. M. 2004, ApJ, 604, 328

Richer, H. B., et al. 1997, ApJ, 484, 741

Robinson, C., Lyne, A. G., Manchester, R. N., Bailes, M., D’Amico, N., \& Johnston, S. 1995, MNRAS, 274, 547

Romani, R. W. 1987, ApJ, 313, 718

Saito, Y., Kawai, N., Kamae, T., Shibata, S., Dotani, T., \& Kulkarni, S. R. 1997, ApJ, 477, L37

Stappers, B. W., Gaensler, B. M., Kaspi, V. M., van der Klis, M., \& Lewin, W. H. G. 2003, Science, 299, 1372

Stappers, B. W., et al. 1996, ApJ, 465, L119

Thompson, D. J. 1996, in IAU Colloq. 160, Pulsars: Problems and Progress, ed. S. Johnston, M. A. Walker, \& M. Bailes (ASP Conf. Ser. 105; San Francisco: ASP), 307

Toscano, M., Bailes, M., Manchester, R., \& Sandhu, J. 1998, ApJ, 506, 863

Toscano, M., Britton, M. C., Manchester, R. N., Bailes, M., Sandhu, J. S., Kulkarni, S. R., \& Anderson, S. B. 1999a, ApJ, 523, L171

Toscano, M., Sandhu, J. S., Bailes, M., Manchester, R. N., Britton, M. C., Kulkarni, S. R., Anderson, S. B., \& Stappers, B. W. 1999b, MNRAS, 307, 925 van Straten, W., Bailes, M., Britton, M., Kulkarni, S. R., Anderson, S. B., Manchester, R. N., \& Sarkissian, J. 2001, Nature, 412, 158

Webb, N. A., Olive, J.-F., \& Barret, D. 2004a, A\&A, 417, 181

Webb, N. A., Olive, J.-F., Barret, D., Kramer, M., Cognard, I., \& Löhmer, O. 2004b, A\&A, 419, 269

Zavlin, V. E., Pavlov, G. G., Sanwal, D., Manchester, R. N., Trümper, J., Halpern, J. P., \& Becker, W. 2002, ApJ, 569, 894

Zavlin, V. E., Pavlov, G. G., \& Shibanov, Yu. A. 1996, A\&A, 315, 141

Zhang, L., \& Cheng, K. S. 2003, A\&A, 398, 639 